\title{
ON THE SPECTRUM OF AN HAMILTONIAN IN FOCK SPACE. DISCRETE SPECTRUM ASYMPTOTICS
}

\author{
SERGIO ALBEVERIO ${ }^{1,2,3}$, SAIDAKHMAT N. LAKAEV ${ }^{4}$, TULKIN H. RASULOV ${ }^{5}$
}

\begin{abstract}
A model operator $H$ associated with the energy operator of a system describing three particles in interaction, without conservation of the number of particles, is considered. The precise location and structure of the essential spectrum of $H$ is described. The existence of infinitely many eigenvalues below the bottom of the essential spectrum of $H$ is proved for the case where an associated generalized Friedrichs model has a resonance at the bottom of its essential spectrum. An asymptotics for the number $N(z)$ of eigenvalues below the bottom of the essential spectrum is also established. The finiteness of eigenvalues of $H$ below the bottom of the essential spectrum is proved if the associated generalized Friedrichs model has an eigenvalue with energy at the bottom of its essential spectrum.
\end{abstract}

Subject Classification: Primary: 81Q10, Secondary: 35P20, 47N50

Key words and phrases: Model operator, conservation of number of particles, eigenvalues, Efimov effect, Birman-Schwinger principle, essential spectrum, Hilbert-Schmidt, infinitely many eigenvalues, generalized Friedrichs model, conditionally negative function.

\section{INTRODUCTION}

The main goal of the present paper is to give a thorough mathematical treatment of the spectral properties for a model operator $H$ with emphasis on the asymptotics for the number of infinitely many eigenvalues (Efimov's effect case). The model operator $H$ is associated with a system describing three particles in interaction, without conservation of the number of particles.

The Efimov effect is one of the remarkable results in the spectral analysis for continuous three-particle Schrödinger operators: if none of the three two-particle Schrödinger operators (corresponding to the two-particle subsystems) has negative eigenvalues, but at least two of them have a zero energy resonance, then this three-particle Schrödinger operator has an infinite number of discrete eigenvalues, accumulating at zero.

Since its discovery by Efimov in $1970[12]$ many works have been devoted to this subject. See, for example [2, 7, 9, 13, 29, 33, 34, 35, 37].

The main result obtained by Sobolev [33] (see also [35]) is an asymptotics of the form $\mathcal{U}_{0}|\log | \lambda||$ for the number of eigenvalues on the left of $\lambda, \lambda<0$, where the coefficient $\mathcal{U}_{0}$ does not depend on the two-particle potentials $v_{\alpha}$ and is a positive function of the ratios $m_{1} / m_{2}, m_{2} / m_{3}$ of the masses of the three-particles.

Recently the existence of the Efimov effect for $N$-body quantum systems with $N \geq 4$ has been proved by X.P. Wang in [36].

In fact in [36] a lower bound on the number of eigenvalues of the total (reduced) Hamiltonian of the form

$$
C_{0}\left|\log \left(E_{0}-\lambda\right)\right|
$$

Date: October 26, 2018. 
is given, when $\lambda$ tends to $E_{0}$, where $C_{0}$ is a positive constant and $E_{0}$ is the bottom of the essential spectrum.

The kinematics of the quantum systems of describing three quasi-particles on lattices is rather exotic. For instance, due to the fact that the discrete analogue of the Laplacian (or its generalizations) is not rotationally invariant, the Hamiltonian of a system does not separate into two parts, one relating to the center-of-mass motion and the other one to the internal degrees of freedom. In particular, the Efimov effect exists only for the zero value of the three-particle quasi-momentum $K \in \mathbb{T}^{3}$ (see, e.g., [3, 4] 6 17 20] 21] 25] for relevant discussions and [10, 11, 16, 25, 26, 28, 31, 38, 40] for the general study of the low-lying excitation spectrum for quantum systems on lattices).

In statistical physics [27] 24], solid-state physics [28] and the theory of quantum fields [15] some important problems arise where the number of quasi-particles is bounded, but not fixed. The study of systems describing $n$ particles in interaction, without conservation of the number of particles is reduced to the investigation of the spectral properties of selfadjoint operators acting in the cut subspace $\mathcal{H}^{(n)}$ of the Fock space, consisting of $r \leq n$ particles [15, 27, 28, 39].

The perturbation problem of an operator (a generalized Friedrichs model), with point and continuous spectrum (which acts in $\mathcal{H}^{(2)}$ ) has played a considerable role in the spectral problems connected with the quantum theory of fields [15].

In the present paper we consider the perturbation problem, with two- and three-particle essential and point spectrum. Under some smoothness assumptions on the parameters of a family of generalized Friedrichs models $h(p), p \in \mathbb{T}^{3}=(-\pi, \pi]^{3}$ we obtain the following results:

(i) We describe the location and structure of the essential spectrum of $H$ via the spectrum of $h(p), p \in \mathbb{T}^{3}$.

(ii) We prove that the operator $H$ has infinitely many eigenvalues below the bottom of the essential spectrum $\sigma_{\text {ess }}(H)$, if the operator $h(0)$ has a resonance with energy at the bottom of its essential spectrum. Moreover, we establish the following asymptotic formula for the number $N(z)$ of eigenvalues of $H$ lying below $z<m=\inf \sigma_{\text {ess }}(H)$

$$
\lim _{z \rightarrow m-0} \frac{N(z)}{|\log | z-m||}=\mathcal{U}_{0}\left(0<\mathfrak{U}_{0}<\infty\right) .
$$

(iii) We prove the finiteness of eigenvalues of $H$ below the bottom of $\sigma_{e s s}(H)$, if $h(0)$ has an eigenvalue with energy at the bottom of its essential spectrum.

We remark that the presence of a zero energy resonance for the Schrödinger operators is due to the two-particle interaction operators $V$, in particular, the coupling constant (if $V$ has in front of it a couling constant) (see, e.g., [1, 19, 30, 37] ).

It is remarkable that for the generalized Friedrichs model $h(0)$ the presence of a resonance with energy at the bottom of its essential spectrum (consequently the existence of infinitely many eigenvalues of $H$ ) is due to the annihilation and creation operators acting in the symmetric Fock space.

We notice that the assertion (iii) is surprising and similar assertions have not yet been proved for the three-particle Schrödinger operators on $\mathbb{R}^{3}$ or $\mathbb{Z}^{3}$.

We remark that the operator $H$ has been considered before, but only the existence of infinitely many eigenvalues below the bottom of the essential spectrum of $H$ has been announced in [22] and only the location of the essential spectrum of $H$ has been described in terms of zeroes of the Friedholm determinant in [23] in the case where the functions $u, v$ and $w$ were analytic. 
The organization of the present paper is as follows. Section 1 is an introduction to the whole work. In Section 2 the model operator is described as a bounded self-adjoint operator $H$ in $\mathcal{H}^{(3)}$ and the main results of the present paper are formulated. Some spectral properties of $h(p), p \in \mathbb{T}^{3}$ are studied in Section 3. In Section 4 the location and structure of the essential spectrum of $H$ is studied. In Section 5 we prove the Birman-Schwinger principle for the operator $H$. In Section 6 the finiteness of the number of eigenvalues of the operator $H$ is established. In Section 7 an asymptotic formula for the number of eigenvalues is proved. Some technical material is collected in Appendix $A$.

Throughout the present paper we adopt the following conventions: Denote by $\mathbb{T}^{3}$ the three-dimensional torus, the cube $(-\pi, \pi]^{3}$ with appropriately identified sides. The torus $\mathbb{T}^{3}$ will always be considered as an abelian group with respect to the addition and multiplication by real numbers regarded as operations on $\mathbb{R}^{3}$ modulo $(2 \pi \mathbb{Z})^{3}$.

For each $\delta>0$ the notation $U_{\delta}(0)=\left\{p \in \mathbb{T}^{3}:|p|<\delta\right\}$ stands for a $\delta$-neighborhood of the origin.

Let $\mathcal{B}\left(\theta, \mathbb{T}^{3}\right)$ with $1 / 2<\theta<1$, be the Banach spaces of Hölder continuous functions on $\mathbb{T}^{3}$ with exponent $\theta$ obtained by the closure of the space of smooth (periodic) functions $f$ on $\mathbb{T}^{3}$ with respect to the norm

$$
\|f\|_{\theta}=\sup _{\substack{t, \ell \in \mathbb{T}^{3} \\ l \neq 0}}\left[|f(t)|+|\ell|^{-\theta}|f(t+\ell)-f(t)|\right] .
$$

The set of functions $f: \mathbb{T}^{3} \rightarrow \mathbb{R}$ having continuous derivatives up to order $n$ inclusive will be denoted $C^{(n)}\left(\mathbb{T}^{3}\right)$. In particular $C^{(0)}\left(\mathbb{T}^{3}\right)=C\left(\mathbb{T}^{3}\right)$ by our convention that $f^{(0)}(x)=f(x)$.

\section{THE MODEL OPERATOR AND STATEMENT OF RESULTS}

Let us introduce some notations used in this work. Let $\mathbb{C}=\mathbb{C}^{1}$ be the field of complex numbers and let $L_{2}\left(\mathbb{T}^{3}\right)$ be the Hilbert space of square-integrable (complex) functions defined on $\mathbb{T}^{3}$ and $L_{2}^{s}\left(\left(\mathbb{T}^{3}\right)^{2}\right)$ be the Hilbert space of square-integrable symmetric (complex) functions on $\left(\mathbb{T}^{3}\right)^{2}$.

Denote by $\mathcal{H}^{(3)}$ the direct sum of spaces $\mathcal{H}_{0}=\mathbb{C}^{1}, \mathcal{H}_{1}=L_{2}\left(\mathbb{T}^{3}\right)$ and $\mathcal{H}_{2}=L_{2}^{s}\left(\left(\mathbb{T}^{3}\right)^{2}\right)$, that is, $\mathcal{H}^{(3)}=\mathcal{H}_{0} \oplus \mathcal{H}_{1} \oplus \mathcal{H}_{2}$.

Let $H$ be the operator in $\mathcal{H}^{(3)}$ with the entries $H_{i j}: \mathcal{H}_{j} \rightarrow \mathcal{H}_{i}, i, j=0,1,2$ :

$$
\begin{gathered}
\left(H_{00} f_{0}\right)_{0}=u_{0} f_{0}, \quad\left(H_{01} f_{1}\right)_{0}=\int_{\mathbb{T}^{3}} v\left(q^{\prime}\right) f_{1}\left(q^{\prime}\right) d q^{\prime}, \quad H_{02}=0, \\
H_{10}=H_{01}^{*}, \quad\left(H_{11} f_{1}\right)_{1}(p)=u(p) f_{1}(p), \quad\left(H_{12} f_{2}\right)_{1}(p)=\int_{\mathbb{T}^{3}} v\left(q^{\prime}\right) f_{2}\left(p, q^{\prime}\right) d q^{\prime}, \\
H_{20}=0, \quad H_{21}=H_{12}^{*}, \quad\left(H_{22} f_{2}\right)_{2}(p, q)=w(p, q) f_{2}(p, q),
\end{gathered}
$$

where $H_{i j}^{*}: \mathcal{H}_{i} \rightarrow \mathcal{H}_{j},(j=i+1, i=0,1)$ denotes the adjoint operator to $H_{i j}$.

Here $f_{i} \in \mathcal{H}_{i}, i=0,1,2, u_{0}$ is a real number, $u$ is a real-valued essentially bounded function on $\mathbb{T}^{3}, v$ is a real-valued function belonging to $L_{2}\left(\mathbb{T}^{3}\right)$ and $w$ is a real-valued essentially bounded symmetric function on $\left(\mathbb{T}^{3}\right)^{2}$.

Under these assumptions the operator $H$ is bounded and self-adjoint in $\mathcal{H}$.

We remark that the operators $H_{10}$ and $H_{21}$ resp. $H_{01}$ and $H_{12}$ defined in the Fock space are called creation resp. annihilation operators.

Throughout this paper we assume the following additional assumptions. 
Assumption 2.1. ( $i)$ The symmetric function $w$ on $\left(\mathbb{T}^{3}\right)^{2}$ is even with respect to $(p, q)$, and has a unique non-degenerate minimum at the point $(0,0) \in\left(\mathbb{T}^{3}\right)^{2}$ and all third order partial derivatives of $w$ belong to $\mathcal{B}\left(\theta,\left(\mathbb{T}^{3}\right)^{2}\right)$.

(ii) There exist positive definite matrix $W$ and real numbers $l_{1}, l_{2}\left(l_{1}>0, l_{2} \neq 0\right)$ such that

$$
\left(\frac{\partial^{2} w(0,0)}{\partial p_{i} \partial p_{j}}\right)_{i, j=1}^{3}=l_{1} W,\left(\frac{\partial^{2} w(0,0)}{\partial p_{i} \partial q_{j}}\right)_{i, j=1}^{3}=l_{2} W .
$$

Assumption 2.2. The function $u \in C^{(2)}\left(\mathbb{T}^{3}\right)$ is even on $\mathbb{T}^{3}$, has a unique non-degenerate minimum at the point $0 \in \mathbb{T}^{3}$ and the function $v \in \mathcal{B}\left(\theta, \mathbb{T}^{3}\right)$, with $1 / 2<\theta<1$, is even.

Remark 2.3. In fact in the present paper we use only the condition $v^{2} \in \mathcal{B}\left(\theta, \mathbb{T}^{3}\right)$.

Remark 2.4. Note that if the function $w$ resp. $u$ is even and has a unique minimum at a point $\left(p_{0}, p_{0}\right) \in\left(\mathbb{T}^{3}\right)^{2}$ resp. $p_{1} \in \mathbb{T}^{3}$, then $p_{0}=0$ resp. $p_{1}=0$. Therefore, without loss of generality we assume that the function $w$ resp. $u$ has a unique minimum at the point $(0,0) \in\left(\mathbb{T}^{3}\right)^{2}$ resp. $0 \in \mathbb{T}^{3}$.

Set

$$
m=\min _{p, q \in \mathbb{T}^{3}} w(p, q), \quad M=\max _{p, q \in \mathbb{T}^{3}} w(p, q)
$$

and

$$
\Lambda(p, z)=\int_{\mathbb{T}^{3}} \frac{v^{2}(t) d t}{w(p, t)-z}, p \in \mathbb{T}^{3}, z \leq m .
$$

Assumption 2.5. Assume that

(i) The function $\Lambda(\cdot, m)$ has a unique maximum at $p=0 \in \mathbb{T}^{3}$.

ii) There exist positive numbers $\delta$ and c such that for all nonzero $p \in U_{\delta}(0)$ the inequality

$$
\Lambda(0, m)-\Lambda(p, m)>c p^{2}
$$

holds.

Remark 2.6. Let $\varepsilon$ be a real-analytic conditionally negative definite function on $\mathbb{T}^{3}$ with a unique non-degenerate minimum at the origin and the function $v \in \mathcal{B}\left(\theta, \mathbb{T}^{3}\right)$ is even and

$$
u(p)=\varepsilon(p)+c, w(p, q)=\varepsilon(p)+\varepsilon(p+q)+\varepsilon(q)
$$

for some real c. Then Assumptions 2.1] 2.2 and 2.5 are fulfilled (see Lemma A.1].

To formulate the main results of the paper we introduce a family of generalized Friedrichs model $h(p), p \in \mathbb{T}^{3}$ which acts in $\mathcal{H}^{(2)} \equiv \mathcal{H}_{0} \oplus \mathcal{H}_{1}$ with the entries

$$
\begin{array}{r}
\left(h_{00}(p) f_{0}\right)_{0}=u(p) f_{0}, h_{01}=\frac{1}{\sqrt{2}} H_{01}, \\
h_{10}=h_{01}^{*},\left(h_{11}(p) f_{1}\right)_{1}(q)=w_{p}(q) f_{1}(q),
\end{array}
$$

where $w_{p}(q)=w(p, q)$.

Let the operator $h_{0}(p), p \in \mathbb{T}^{3}$ act in $\mathcal{H}^{(2)}$ as

$$
h_{0}(p)\left(\begin{array}{l}
f_{0} \\
f_{1}(q)
\end{array}\right)=\left(\begin{array}{l}
0 \\
w_{p}(q) f_{1}(q)
\end{array}\right) .
$$

The perturbation $h(p)-h_{0}(p)$ of the operator $h_{0}(p)$ is a self-adjoint operator of rank 2 . Therefore in accordance with the invariance of the essential spectrum under finite rank 
perturbations the essential spectrum $\sigma_{\text {ess }}(h(p))$ of $h(p)$ fills the following interval on the real axis:

$$
\sigma_{\text {ess }}(h(p))=[m(p), M(p)],
$$

where the numbers $m(p)$ and $M(p)$ are defined by

$$
m(p)=\min _{q \in \mathbb{T}^{3}} w(p, q) \quad \text { and } \quad M(p)=\max _{q \in \mathbb{T}^{3}} w(p, q) .
$$

Remark 2.7. We remark that for some $p \in \mathbb{T}^{3}$ the essential spectrum of $h(p)$ may degenerate to the set consisting of unique point $[m(p), m(p)]$ and hence we can not state that the essential spectrum of $h(p)$ is absolutely continuous for any $p \in \mathbb{T}^{3}$. For example, if the function $w$ is of the form (2.1), where

$$
\varepsilon(q)=3-\cos q_{1}-\cos q_{2}-\cos q_{3}, \quad q=\left(q_{1}, q_{2}, q_{3}\right) \in \mathbb{T}^{3}
$$

and $q=(\pi, \pi, \pi) \in \mathbb{T}^{3}$.

The following theorem describes the essential spectrum of the operator $H$.

Theorem 2.8. For the essential spectrum $\sigma_{\text {ess }}(H)$ of the operator $H$ the equality

$$
\sigma_{e s s}(H)=\cup_{p \in \mathbb{T}^{3}} \sigma_{d}(h(p)) \cup[m, M]
$$

holds, where $\sigma_{d}(h(p))$ is the discrete spectrum of the operator $h(p), p \in \mathbb{T}^{3}$.

For any $p \in \mathbb{T}^{3}$ we define an analytic function (the Fredholm determinant associated with the operator $h(p)) \Delta(p, z)$ in $\mathbb{C} \backslash[m(p), M(p)]$ by

$$
\Delta(p, z)=u(p)-z-\frac{1}{2} \int_{\mathbb{T}^{3}} \frac{v^{2}(q) d q}{w_{p}(q)-z} .
$$

Let $\sigma$ be the set of all complex numbers $z \in \mathbb{C} \backslash[m(p), M(p)]$ such that the equality $\Delta(p, z)=0$ holds for some $p \in \mathbb{T}^{3}$.

Remark 2.9. We remark that in [23] the essential spectrum of the operator $H$ has been described by means of zeroes of the Fredholm determinant defined in 2.3. and by the spectrum of multiplication operator $\mathrm{H}_{22}$ as follows:

$$
\sigma_{\text {ess }}(H)=\sigma \cup[m, M] .
$$

We note that the equality

$$
\sigma=\cup_{p \in \mathbb{T}^{3}} \sigma_{d}(h(p))
$$

holds (see Lemma 4.2).

Definition 2.10. The set $\sigma$ resp. $[m, M]$ is called two- resp. three-particle branch of the essential spectrum $\sigma_{\text {ess }}(H)$ of the operator $H$, which will be denoted by $\sigma_{\text {two }}(H)$ resp. $\sigma_{\text {three }}(H)$.

The function $w_{0}(\cdot)$ has a unique non-degenerate minimum at $q=0$ (see Lemma A.2 and hence by Lebesgue's dominated convergence theorem the finite limit

$$
\Delta(0, m)=\lim _{z \rightarrow m-0} \Delta(0, z)
$$

exists. 
Definition 2.11. Let part (i) of Assumption 2.1] be fulfilled and $u(0) \neq m$. The operator $h(0)$ is said to have an menergy resonance if the number 1 is an eigenvalue of the operator

$$
(\mathrm{G} \psi)(q)=\frac{v(q)}{2(u(0)-m)} \int_{\mathbb{T}^{3}} \frac{v(t) \psi(t) d t}{w_{0}(t)-m}, \psi \in C\left(\mathbb{T}^{3}\right)
$$

and the associated eigenfunction $\psi$ (up to a constant factor) satisfies the condition $\psi(0) \neq$ 0 .

Remark 2.12. Let part ( $i$ ) of Assumption 2.1 be fulfilled and $v \in \mathcal{B}\left(\theta, \mathbb{T}^{3}\right)$.

(i) If $u(0) \leq m$, then the equation $h(0) f=m f$ has only the trivial solution $f \in \mathbb{C}^{1} \oplus$ $L_{1}\left(\mathbb{T}^{3}\right)$.

(ii) Assume that $u(0)>m$ and $\Delta(0, m)=0$.

a) If $v(0) \neq 0$, then the operator $h(0)$ has an $m$ energy resonance and the vector $f=$ $\left(f_{0}, f_{1}\right)$, where

$$
f_{0}=\text { const } \neq 0, f_{1}(q)=-\frac{v(q) f_{0}}{\sqrt{2}\left(w_{0}(q)-m\right)},
$$

obeys the equation $h(0) f=m f$ and hence $f_{1} \in L_{1}\left(\mathbb{T}^{3}\right) \backslash L_{2}\left(\mathbb{T}^{3}\right)$ (see Lemma 3.2).

b) If $v(0)=0$, then the number $z=m$ is an eigenvalue of the operator $h(0)$ and the vector $f=\left(f_{0}, f_{1}\right)$, where $f_{0}$ and $f_{1}$ are defined by (2.4), obeys the equation $h(0) f=m f$ and hence $f_{1} \in L_{2}\left(\mathbb{T}^{3}\right)$ (see Lemma 3.3).

Let us denote by $\tau_{\text {ess }}(H)$ the bottom of the essential spectrum $\sigma_{\text {ess }}(H)$ of the operator $H$ and by $N(z)$ the number of eigenvalues of $H$ lying below $z \leq \tau_{\text {ess }}(H)$.

The main result of this paper is the following

Theorem 2.13. Let Assumptions 2.1 and 2.2 be fulfilled.

(i) Assume that the number $z=m$ is an eigenvalue of $h(0)$ and let Assumption 2.5 be fulfilled. Then the operator $H$ has a finite number of eigenvalues lying below $\tau_{\text {ess }}(H)=$ m.

(ii) Assume that the operator $h(0)$ has an menergy resonance and assume that part (i) of Assumption 2.5 is fulfilled. Then the operator $H$ has infinitely many eigenvalues lying below $\tau_{\text {ess }}(H)=m$ and accumulating at $\tau_{\text {ess }}(H)=m$. Moreover the function $N(\cdot)$ obeys the relation

$$
\lim _{z \rightarrow m-0} \frac{N(z)}{|\log | z-m||}=\mathfrak{U}_{0}\left(0<\mathfrak{U}_{0}<\infty\right) .
$$

Remark 2.14. The constant $U_{0}$ does not depend on the function $v$ and is given as a positive function depending only on the ratio $\frac{l_{1}}{l_{2}}$ (with $l_{1}, l_{2}$ as in Assumption [2.I].

Remark 2.15. We remark that if the conditions of Theorem 2.13] are fulfilled, then $\inf \sigma_{\text {ess }}(H)=m$ (see Lemma A.3.

Remark 2.16. We remark that in [4] a result which is an analogue of part (ii) of Theorem 2.13 has been proven for the three-particle Schrödinger operators associated with a system of three-particles on lattices interacting by means zero-range pair potentials.

Remark 2.17. Clearly, the infinite cardinality of the discrete spectrum of $H$ lying on the l.h.s. of $m$ follows automatically from the positivity of $\mathfrak{U}_{0}$. 


\section{SPECTRAL PROPERTIES OF THE OPERATORS $h(p), p \in \mathbb{T}^{3}$}

In this section we study some spectral properties of the family of generalized Friedrichs model $h(p), p \in \mathbb{T}^{3}$ given by (2.2), which plays a crucial role in the study of the spectral properties of $H$. We notice that the spectrum and resonances of a generalized Friedrichs model have been studied in detail in [5] [18].

Lemma 3.1. For any $p \in \mathbb{T}^{3}$ the operator $h(p)$ has an eigenvalue $z \in \mathbb{C} \backslash[m(p), M(p)]$ if and only if $\Delta(p, z)=0$.

Proof. If $u(p) \in \mathbb{R} \backslash[m(p), M(p)]$ for any $p \in \mathbb{T}^{3}$, then the equation $h(p) f=m(p) f, f \in$ $\mathcal{H}^{(2)}$ has only trivial solution and hence the value $u(p) \in \mathbb{R} \backslash[m(p), M(p)]$ can not be an eigenvalue of the operator $h(p)$, where $\mathbb{R}$ is the set of real numbers. Therefore, the number $z \in \mathbb{C} \backslash([m(p), M(p)] \cup\{u(p)\})$ is an eigenvalue of the operator $h(p), p \in \mathbb{T}^{3}$ if and only if (by the Birman-Schwinger principle) the number 1 is an eigenvalue of the operator

$$
(\mathrm{G}(p, z) \psi)(q)=\frac{v(q)}{2(u(p)-z)} \int_{\mathbb{T}^{3}} \frac{v(t) \psi(t) d t}{w_{p}(t)-z}, \psi \in L_{2}\left(\mathbb{T}^{3}\right) .
$$

According to the Fredholm theorem the number $\lambda=1$ is an eigenvalue of the operator $G(p, z)$ if and only if $\Delta(p, z)=0$.

Lemma 3.2. Let part $(i)$ of Assumption 2.1 be fulfilled. The operator $h(0)$ has an $m$ energy resonance if and only if $\Delta(0, m)=0$ and $v(0) \neq 0$.

Proof. "Only If Part". Suppose that the operator $h(0)$ has an $m$ energy resonance. Then by Definition 2.11 the inequality $u(0) \neq m$ holds and the equation

$$
\psi(q)=\frac{v(q)}{2(u(0)-m)} \int_{\mathbb{T}^{3}} \frac{v(t) \psi(t) d t}{w_{0}(t)-m}, \psi \in C\left(\mathbb{T}^{3}\right)
$$

has a nontrivial solution $\psi \in C\left(\mathbb{T}^{3}\right)$ which satisfies the condition $\psi(0) \neq 0$.

This solution is equal to the function $v$ (up to a constant factor) and hence

$$
\Delta(0, m)=u(0)-m-\frac{1}{2} \int_{\mathbb{T}^{3}} \frac{v^{2}(t) d t}{w_{0}(t)-m}=0 .
$$

"If Part". Let the equality $\Delta(0, m)=0$ holds and $v(0) \neq 0$. Then the inequality $u(0) \neq m$ holds and the function $v \in C\left(\mathbb{T}^{3}\right)$ is a solution of the equation 3.1), that is, by Definition 2.11 the operator $h(0)$ has an $m$ energy resonance.

Lemma 3.3. Let part $(i)$ of Assumption 2.1] be fulfilled and $v \in \mathcal{B}\left(\theta, \mathbb{T}^{3}\right)$. The number $z=m$ is an eigenvalue of the operator $h(0)$ if and only if $\Delta(0, m)=0$ and $v(0)=0$.

Proof. "Only If Part". Suppose $f=\left(f_{0}, f_{1}\right)$ is an eigenvector of the operator $h(0)$ associated with the eigenvalue $z=m$. Then $f_{0}$ and $f_{1}$ satisfy the system of equations

$$
\left\{\begin{array}{l}
(u(0)-m) f_{0}+\frac{1}{\sqrt{2}} \int_{\mathbb{T}^{3}} v\left(q^{\prime}\right) f_{1}\left(q^{\prime}\right) d q^{\prime}=0 \\
\frac{1}{\sqrt{2}} v(q) f_{0}+\left(w_{0}(q)-m\right) f_{1}(q)=0
\end{array}\right.
$$

From (3.2) we find that $f_{0}$ and $f_{1}$ are given by (2.4) and from the first equation of (3.2) we derive the equality $\Delta(0, m)=0$.

Since $w_{0}(\cdot) \in C^{(3)}\left(\mathbb{T}^{3}\right)$ and $v(\cdot) \in \mathcal{B}\left(\theta, \mathbb{T}^{3}\right)$ and the function $w_{0}(\cdot)$ has a unique non-degenerate minimum at the origin we conclude that $f_{1} \in L_{2}\left(\mathbb{T}^{3}\right)$ iff $v(0)=0$. 
"If Part". Let $v(0)=0$ and $\Delta(0, m)=0$. Then the vector $f=\left(f_{0}, f_{1}\right)$, where $f_{0}$ and $f_{1}$ are defined by (2.4), obeys the equation $h(0) f=m f$ and $f_{1} \in L_{2}\left(\mathbb{T}^{3}\right)$.

Let $v(\cdot) \in \mathcal{B}\left(\theta, \mathbb{T}^{3}\right)$. For any $p, q \in \mathbb{T}^{3}$ and $z<m$ the inequality $w_{p}(q)-z>0$ implies the inequality

$$
\int_{\mathbb{T}^{3}} \frac{v^{2}(t) d t}{w_{p}(t)-z}>0 .
$$

Since the function $w(\cdot, \cdot)$ has a unique non-degenerate minimum at the point $(0,0) \in$ $\left(\mathbb{T}^{3}\right)^{2}$ the integral

$$
\int_{\mathbb{T}^{3}} \frac{v^{2}(t) d t}{w_{p}(t)-m}
$$

is finite. The Lebesgue dominated convergence theorem yields the equality

$$
\Delta(0, m)=\lim _{p \rightarrow 0} \Delta(p, m)
$$

and hence the function $\Delta(\cdot, m)$ is continuous on $\mathbb{T}^{3}$.

Lemma 3.4. Let part (i) of Assumption 2.1] be fulfilled.

(i) Assume that $\max _{p \in \mathbb{T}^{3}} \Delta(p, m)<0$. Then for any $p \in \mathbb{T}^{3}$ the operator $h(p)$ has a unique eigenvalue lying on the l.h.s. of $m$.

(ii) Assume that $\min _{p \in \mathbb{T}^{3}} \Delta(p, m)<0$ and $\max _{p \in \mathbb{T}^{3}} \Delta(p, m) \geq 0$. Then there exists a non void open set $D \subset \mathbb{T}^{3}$ such that $D \neq \mathbb{T}^{3}$ and for any $p \in D$ the operator $h(p)$ has a unique eigenvalue lying on the l.h.s. of $m$ and for any $p \in \mathbb{T}^{3} \backslash D$ the operator $h(p)$ has no eigenvalues lying on the l.h.s. of $m$.

(iii) Assume that $\min _{p \in \mathbb{T}^{3}} \Delta(p, m) \geq 0$. Then for any $p \in \mathbb{T}^{3}$ the operator $h(p)$ has no eigenvalues lying on the l.h.s. of $m$.

Proof. First we prove part $(i i)$.

Let $\min _{p \in \mathbb{T}^{3}} \Delta(p, m)<0$ and $\max _{p \in \mathbb{T}^{3}} \Delta(p, m) \geq 0$.

Introduce the notation

$$
D \equiv\left\{p \in \mathbb{T}^{3}: \Delta(p, m)<0\right\} .
$$

Since $\mathbb{T}^{3}$ is compact and the function $\Delta(\cdot, m)$ is continuous on $\mathbb{T}^{3}$, there exist points $p_{0}, p_{1} \in \mathbb{T}^{3}$ such that the inequalities

$$
\min _{p \in \mathbb{T}^{3}} \Delta(p, m)=\Delta\left(p_{0}, m\right)<0 \text { and } \max _{p \in \mathbb{T}^{3}} \Delta(p, m)=\Delta\left(p_{1}, m\right) \geq 0
$$

hold. Hence we have that $D$ is a non void open set and $D \neq \mathbb{T}^{3}$.

For any $p \in \mathbb{T}^{3}$ the function $\Delta(p, \cdot)$ is continuous and decreasing on $(-\infty, m]$ and

$$
\lim _{z \rightarrow-\infty} \Delta(p, z)=+\infty
$$

Then for any $p \in D$ there exist a unique point $z(p) \in(-\infty, m)$ such that $\Delta(p, z(p))=$ 0 . By Lemma 3.1 for any $p \in D$ the point $z(p)$ is the unique eigenvalue of the operator $h(p)$ lying on the 1.h.s. of $m$.

For any $p \in \mathbb{T}^{3} \backslash D$ and $z<m$ we have

$$
\Delta(p, z)>\Delta(p, m) \geq 0 .
$$

Hence by Lemma 3.1 for each $p \in \mathbb{T}^{3} \backslash D$ the operator $h(p)$ has no eigenvalue lying on the 1.h.s. of $m$. 
If $\max _{p \in \mathbb{T}^{3}} \Delta(p, m)<0$ (resp. $\min _{p \in \mathbb{T}^{3}} \Delta(p, m) \geq 0$ ), then $D=\mathbb{T}^{3}$ (resp. $D=\emptyset$ ) and the above analysis leads again to the case $(i)$ (resp. (iii)). The straightforward details are omitted.

Set

$$
\mathbb{C}_{+}=\{z \in \mathbb{C}: \operatorname{Re} z>0\}, \quad \mathbb{R}_{+}=\{x \in \mathbb{R}: x>0\}, \quad \mathbb{R}_{+}^{0}=\mathbb{R}_{+} \cup\{0\} .
$$

Let $w_{0}(\cdot, \cdot)$ be the function defined on $U_{\delta}(0) \times \mathbb{T}^{3}, \delta>0$ sufficiently small, as

$$
w_{0}(p, q)=w_{p}\left(q+q_{0}(p)\right)-m(p)
$$

where $q_{0}(\cdot) \in C^{(3)}\left(U_{\delta}(0)\right)$ and for any $p \in U_{\delta}(0)$ the point $q_{0}(p)$ is the non-degenerate minimum of the function $w_{p}(\cdot)$ (see Lemma A.2. Here $C^{(n)}\left(U_{\delta}(0)\right)$ can be defined similarly to $C^{(n)}\left(\mathbb{T}^{3}\right)$.

For any $p \in U_{\delta}(0)$ we define an analytic function $D(p, \zeta)$ in $\mathbb{C}_{+}$by

$$
D(p, \zeta)=u(p)-m(p)+\zeta^{2}-\frac{1}{2} \int_{\mathbb{T}^{3}} \frac{v^{2}\left(q+q_{0}(p)\right) d q}{w_{0}(p, q)+\zeta^{2}} .
$$

Lemma 3.5. Let Assumptions 2.1] and 2.2] be fulfilled. Then there exist a number $\delta>0$ such that

i) For any $\zeta \in \mathbb{C}_{+}$the function $D(\cdot, \zeta)$ is of class $C^{(2)}\left(U_{\delta}(0)\right)$ and the following decomposition

$$
D(p, \zeta)=D(0, \zeta)+D^{r e s}(p, \zeta)
$$

holds, where $D^{\text {res }}(p, \zeta)=O\left(p^{2}\right)$ as $p \rightarrow 0$ uniformly in $\zeta \in \mathbb{R}_{+}^{0}$.

ii) The right-hand derivative of $D(0, \cdot)$ at $\zeta=0$ exists and

$$
D(0, \zeta)=D(0,0)+2 \sqrt{2} \pi^{2} v^{2}(0) l_{1}^{-\frac{3}{2}}(\operatorname{det} W)^{-\frac{1}{2}} \zeta+D^{r e s}(\zeta),
$$

where $D^{\text {res }}(\zeta)=O\left(\zeta^{1+\theta}\right), \zeta \in \mathbb{R}_{+}^{0}$.

Remark 3.6. An analogue lemma has been proven in [4] in the case where the functions $u(\cdot), b(\cdot)$ and $w(\cdot, \cdot)$ are analytic on $\mathbb{T}^{3}$ and $\left(\mathbb{T}^{3}\right)^{2}$, respectively.

Proof. i) Since $m(\cdot) \in C^{(3)}\left(U_{\delta}(0)\right)$ by definition of the function $D(\cdot, \cdot)$ and Assumptions 2.1 and 2.2 we obtain that the function $D(\cdot, \zeta)$ is of class $C^{(2)}\left(U_{\delta}(0)\right)$ for any $\zeta \in \mathbb{C}_{+}$.

Using

$$
w_{0}(p, q)=\frac{l_{1}}{2}(W q, q)+o\left(|p||q|^{2}\right)+o\left(|q|^{2}\right) \text { as }|p|,|q| \rightarrow 0
$$

we obtain that there exists $C>0$ such that for any $\zeta \in \mathbb{R}_{+}^{0}$ and $i, j=1,2,3$ the inequalities

$$
\left|\frac{\partial^{2}}{\partial p_{i} \partial p_{j}} \frac{1}{w_{0}(p, q)+\zeta^{2}}\right| \leq \frac{C}{q^{2}}, p, q \in U_{\delta}(0)
$$

and

$$
\left|\frac{\partial^{2}}{\partial p_{i} \partial p_{j}} \frac{1}{w_{0}(p, q)+\zeta^{2}}\right| \leq C, p \in U_{\delta}(0), q \in \mathbb{T}^{3} \backslash U_{\delta}(0)
$$

hold.

The Lebesgue dominated convergence theorem implies that

$$
\frac{\partial^{2}}{\partial p_{i} \partial p_{j}} D(p, 0)=\lim _{\zeta \rightarrow 0+} \frac{\partial^{2}}{\partial p_{i} \partial p_{j}} D(p, \zeta) .
$$


Repeating the application of the Hadamard lemma (see [41] V.1, p. 512) we obtain

$$
D(p, \zeta)=D(0, \zeta)+\sum_{i=1}^{3} \frac{\partial}{\partial p_{i}} D(0, \zeta) p_{i}+\sum_{i, j=1}^{3} H_{i j}(p, \zeta) p_{i} p_{j},
$$

where for any $\zeta \in \mathbb{R}_{+}^{0}$ the functions $H_{i j}(\cdot, \zeta), i, j=1,2,3$ are continuous in $U_{\delta}(0)$ and

$$
H_{i j}(p, \zeta)=\frac{1}{2} \int_{0}^{1} \int_{0}^{1} \frac{\partial^{2}}{\partial p_{i} \partial p_{j}} D\left(x_{1} x_{2} p, \zeta\right) d x_{1} d x_{2}
$$

The estimates 3.5 and 3.6 give

$$
\left|H_{i, j}(p, \zeta)\right| \leq \frac{1}{2} \int_{0}^{1} \int_{0}^{1}\left|\frac{\partial^{2}}{\partial p_{i} \partial p_{j}} D\left(x_{1} x_{2} p, \zeta\right)\right| d x_{1} d x_{2} \leq C\left(1+\int_{U_{\delta}(0)} \frac{v^{2}\left(q+q_{0}(p)\right) d q}{q^{2}}\right)
$$

for any $p \in U_{\delta}(0)$ uniformly in $\zeta \in \mathbb{R}_{+}^{0}$.

Since for any $\zeta \in \mathbb{C}_{+}$the function $D(\cdot, \zeta)$ is even in $U_{\delta}(0)$ we have

$$
\left[\frac{\partial}{\partial p_{i}} D(p, \zeta)\right]_{p=0}=0, \quad i=1,2,3 .
$$

ii) Now we prove that there exists the right-hand derivative of $D(0, \cdot)$ at $\zeta=0$ and the following inequalities

$$
\begin{gathered}
|D(0, \zeta)-D(0,0)| \leq C \zeta, \quad \zeta \in \mathbb{R}_{+}^{0}, \\
\left|\frac{\partial}{\partial \zeta} D(0, \zeta)-\frac{\partial}{\partial \zeta} D(0,0)\right|<C \zeta^{\theta}, \quad \zeta \in \mathbb{R}_{+}^{0}
\end{gathered}
$$

hold for some positive $C$.

Indeed, the function $D(0, \cdot)$ can be represented as

$$
D(0, \zeta)=D_{1}(\zeta)+D_{2}(\zeta)
$$

with

$$
D_{1}(\zeta)=-\frac{1}{2} \int_{U_{\delta}(0)} \frac{v^{2}(q)}{w_{0}(0, q)+\zeta^{2}} d q, \zeta \in \mathbb{C}_{+}
$$

and

$$
D_{2}(\zeta)=u(0)-m+\zeta^{2}-\frac{1}{2} \int_{\mathbb{T}^{3} \backslash U_{\delta}(0)} \frac{v^{2}(q)}{w_{0}(0, q)+\zeta^{2}} d q, \zeta \in \mathbb{C}_{+} .
$$

Since the function $w_{0}(0, \cdot)$ is continuous on the compact set $\mathbb{T}^{3} \backslash U_{\delta}(0)$ and has a unique minimum at $q=0$ there exists $M>0$ such that $\left|w_{0}(0, q)\right|>M$ for all $q \in \mathbb{T}^{3} \backslash U_{\delta}(0)$.

Then by $v(\cdot) \in \mathcal{B}\left(\theta, \mathbb{T}^{3}\right)$ we have

$$
\left|D_{2}(\zeta)-D_{2}(0)\right| \leq C \zeta^{2}, \zeta \in \mathbb{R}_{+}^{0}
$$

for some $C=C(\delta)>0$.

Applying the Morse lemma and computing some integrals we obtain that (see Lemma A.5 there exists a right-hand derivative of $D_{1}(\cdot)$ at $\zeta=0$ and

$$
\frac{\partial}{\partial \zeta} D_{1}(0)=\lim _{\zeta \rightarrow 0+} \frac{D_{1}(\zeta)-D_{1}(0)}{\zeta}=2 \sqrt{2} \pi^{2} l_{1}^{-\frac{3}{2}} v^{2}(0)(\operatorname{det} W)^{-\frac{1}{2}}
$$

and hence

$$
\left|D_{1}(\zeta)-D_{1}(0)\right|<C \zeta, \quad \zeta \in \mathbb{R}_{+}^{0}
$$

holds for some positive $C$. 
Then from (3.10) and (3.12) it follows that the right-hand derivative of $D(0, \cdot)$ at $\zeta=0$ exists and

$$
\frac{\partial}{\partial \zeta} D(0,0)=2 \sqrt{2} \pi^{2} l_{1}^{-\frac{3}{2}} v^{2}(0)(\operatorname{det} W)^{-\frac{1}{2}} .
$$

Comparing (3.10) and 3.12) we obtain (3.7).

In the same way one can prove the inequality 3.8 .

The following decomposition plays a important role in the proof of the the main result, that is, the asymptotics 2.5.

Corollary 3.7. Let the operator $h(0)$ has an $m$ energy resonance. Then for any $p \in$ $U_{\delta}(0), \delta>0$ sufficiently small, and $z \leq m$ the following decomposition

$$
\Delta(p, z)=2 \sqrt{2} \pi^{2} v^{2}(0) l_{1}^{-\frac{3}{2}}(\operatorname{det} W)^{-\frac{1}{2}} \sqrt{m(p)-z}+\Delta^{(02)}(m(p)-z)+\Delta^{(20)}(p, z)
$$

holds, where $\Delta^{(02)}(m(p)-z)\left(\right.$ resp. $\left.\Delta^{(20)}(p, z)\right)$ is a function behaving like

$O\left((m(p)-z)^{\frac{1+\theta}{2}}\right)$ (resp. $\left.O\left(|p|^{2}\right)\right)$ as $|m(p)-z| \rightarrow 0$ (resp. $p \rightarrow 0$ uniformly in $\left.z \leq m\right)$.

Proof. By Lemma 3.2 we have that $\Delta(0, m)=0$ and $v(0) \neq 0$ and hence the proof of Corollary 3.7 immediately follows from Lemma 3.5 and equality

$\Delta(p, z)=D(p, \sqrt{m(p)-z})$.

Lemma 3.8. Let the operator $h(0)$ has an $m$ energy resonance. Then there exist positive numbers $c, C$ and $\delta$ such that

$$
c|p| \leq \Delta(p, m) \leq C|p| \quad \text { for any } \quad p \in U_{\delta}(0)
$$

and

$$
\Delta(p, m) \geq c \quad \text { for any } \quad p \in \mathbb{T}^{3} \backslash U_{\delta}(0) .
$$

Proof. Corollary 3.7 and the asymptotics (see part (ii) of Lemma A.2

$$
m(p)=m+\frac{l_{1}^{2}-l_{2}^{2}}{2 l_{1}}(W p, p)+o\left(p^{3}\right) \quad \text { as } \quad p \rightarrow 0
$$

yields 3.13 for some positive numbers $c, C$.

The inequality (3.14) follows from the positivity (see proof of Lemma A.3) and continuity of the function $\Delta(\cdot, m)$ on the compact set $\mathbb{T}^{3} \backslash U_{\delta}(0)$.

Lemma 3.9. Let the number $z=m$ is an eigenvalue of the operator $h(0)$. Then there exist numbers $\delta>0$ and $c>0$ such that

$$
\begin{array}{r}
|\Delta(p, m)| \geq c p^{2} \quad \text { for any } \quad p \in U_{\delta}(0) \\
|\Delta(p, m)| \geq c \quad \text { for all } \quad p \in \mathbb{T}^{3} \backslash U_{\delta}(0) .
\end{array}
$$

Proof. By Lemma3.3 we have $\Delta(0, m)=0$ and $v(0)=0$. Then the function $\Delta(\cdot, m)$ can be represented in the form

$$
\Delta(p, m)=u(p)-u(0)+\frac{1}{2}(\Lambda(0, m)-\Lambda(p, m)) .
$$

Then from Assumptions 2.2 and 2.5 it follows that there exist positive numbers $\delta$ and $c$ such that the statement of the lemma is fulfilled. 


\section{THE ESSENTIAL SPECTRUM OF THE OPERATOR $H$}

4.1. The spectrum of the operator $\hat{H}$. We consider the operator $\hat{H}$ acting in $\hat{\mathcal{H}}=L_{2}\left(\mathbb{T}^{3}\right) \oplus L_{2}\left(\left(\mathbb{T}^{3}\right)^{2}\right)$ as

$$
\hat{H}\left(\begin{array}{l}
f_{1}(p) \\
f_{2}(p, q)
\end{array}\right)=\left(\begin{array}{l}
u(p) f_{1}(p)+\frac{1}{\sqrt{2}} \int_{\mathbb{T}^{3}} v\left(q^{\prime}\right) f_{2}\left(p, q^{\prime}\right) d q^{\prime} \\
\frac{1}{\sqrt{2}} v(q) f_{1}(p)+w_{p}(q) f_{2}(p, q)
\end{array}\right) .
$$

The operator $\hat{H}$ commutes with any multiplication operator $U_{\gamma}$ acting in $\hat{\mathcal{H}}$ as

$$
U_{\gamma}\left(\begin{array}{l}
f_{1}(p) \\
f_{2}(p, q)
\end{array}\right)=\left(\begin{array}{l}
\gamma(p) f_{1}(p) \\
\gamma(p) f_{2}(p, q)
\end{array}\right), \gamma \in L_{2}\left(\mathbb{T}^{3}\right) .
$$

Therefore the decomposition of the space $\hat{\mathcal{H}}$ into the direct integral

$$
\hat{\mathcal{H}}=\int_{\mathbb{T}^{3}} \oplus \mathcal{H}^{(2)} d p
$$

yields the decomposition into the direct integral

$$
\hat{H}=\int_{\mathbb{T}^{3}} \oplus h(p) d p
$$

where the fiber operators $h(p), p \in \mathbb{T}^{3}$ are defined by 2.2.

Lemma 4.1. For the spectrum $\sigma(\hat{H})$ of $\hat{H}$ the equality

$$
\sigma(\hat{H}) \equiv \cup_{p \in \mathbb{T}^{3}} \sigma_{d}(h(p)) \cup[m, M]
$$

holds.

Proof. The assertion of the lemma follows from the representation (4.1) of the operator $\hat{H}$ and the theorem on decomposable operators (see [32]).

Lemma 4.2. The essential spectrum $\sigma_{\text {ess }}(H)$ of the operator $H$ coincides with the spectrum of $\hat{H}$, that is,

$$
\sigma_{\text {ess }}(H)=\sigma(\hat{H}) .
$$

Proof. In [23] it has been proved that the essential spectrum $\sigma_{\text {ess }}(H)$ of the operator $H$ coincides with $\sigma \cup[m, M]$. By Lemma3.1 we have

$$
\sigma=\cup_{p \in \mathbb{T}^{3}} \sigma_{d}(h(p))
$$

and hence by Lemma4.1 we obtain 4.2.

4.2. The structure of the essential spectrum of $H$. Let us introduce the following notations:

$$
\sigma_{t w o}(\hat{H})=\cup_{p \in \mathbb{T}^{3}} \sigma_{d}(h(p)), \quad a \equiv \inf \sigma_{t w o}(\hat{H}), \quad b \equiv \sup \sigma_{t w o}(\hat{H}) .
$$

Since for any $p \in \mathbb{T}^{3}$ the function $\Delta(p, \cdot)$ is decreasing in $(M,+\infty)$ by Lemma 3.1 the operator $h(p), p \in \mathbb{T}^{3}$ either has a unique eigenvalue in $(M,+\infty)$ or not. It is easy to see that if $\Delta(p, M) \leq 0$ for all $p \in \mathbb{T}^{3}$, then the operator $h(p), p \in \mathbb{T}^{3}$ has no eigenvalue in $(M,+\infty)$.

Thus the location and structure of the essential spectrum of $H$ can be precisely described as well as in the following 
Theorem 4.3. Let part ( $i$ ) of Assumption 2.1] and Assumption 2.2 be fulfilled and $\Delta(p, M) \leq$ 0 for all $p \in \mathbb{T}^{3}$.

(i) Assume that $\max _{p \in \mathbb{T}^{3}} \Delta(p, m)<0$. Then

$$
\sigma_{\text {ess }}(H)=[a, b] \cup[m, M] \text { and } b<m .
$$

(ii) Assume that $\min _{p \in \mathbb{T}^{3}} \Delta(p, m)<0$ and $\max _{p \in \mathbb{T}^{3}} \Delta(p, m) \geq 0$. Then

$$
\sigma_{\text {ess }}(H)=[a, M] \text { and } a<m .
$$

(iii) Assume that $\min _{p \in \mathbb{T}^{3}} \Delta(p, m) \geq 0$. Then

$$
\sigma_{e s s}(H)=[m, M]
$$

Proof. (i) Let $\max _{p \in \mathbb{T}^{3}} \Delta(p, m)<0$. Then by Lemma 3.4 for all $p \in \mathbb{T}^{3}$ the operator $h(p)$ has a unique eigenvalue $z(p)<m$.

By part $(i)$ of Assumption 2.1 and Assumption 2.2 and Lemma 3.1 the map $z: p \in$ $\mathbb{T}^{3} \rightarrow z(p)$ is of class $C^{(2)}\left(\mathbb{T}^{3}\right)$.

Therefore the range $\operatorname{Im} z$ of the function $z(\cdot)$ is a connected closed subset of $(-\infty, m)$, that is, $\operatorname{Im} z=[a, b]$ and $b<m$ and hence $\sigma_{t w o}(\hat{H})=[a, b]$.

(ii) Let $\min _{p \in \mathbb{T}^{3}} \Delta(p, m)<0$ and $\max _{p \in \mathbb{T}^{3}} \Delta(p, m) \geq 0$. Then by assertion $(i i)$ of Lemma 3.4 there exists a non void open set $D$ such that for any $p \in D$ the operator $h(p)$ has a unique eigenvalue $z(p)<m$.

Since for any $p \in \mathbb{T}^{3}$ the operator $h(p)$ is bounded and $\mathbb{T}^{3}$ is compact set, there exists a positive number $C$ such that $\sup _{p \in \mathbb{T}^{3}}\|h(p)\| \leq C$ and for any $p \in \mathbb{T}^{3}$ we have

$$
\sigma(h(p)) \subset[-C, C] .
$$

For any $q \in \partial D=\left\{p \in \mathbb{T}^{3}: \Delta(p, m)=0\right\}$ there exist $\left\{p_{n}\right\} \subset D$ such that $p_{n} \rightarrow q$ as $n \rightarrow \infty$. Set $z_{n}=z\left(p_{n}\right)$. Then by Lemma 3.4 for any $p_{n} \in D$ the inequality $z_{n}<m$ holds and from (4.4) we get $\left\{z_{n}\right\} \subset[-C, m]$. Without loss of generality (otherwise we would have to take a subsequence) we assume that $z_{n} \rightarrow z_{0}$ as $n \rightarrow \infty$ for some $z_{0} \in[-C, m]$.

From the continuity of the function $\Delta(\cdot, \cdot)$ in $\mathbb{T}^{3} \times(-\infty, m]$ and $p_{n} \rightarrow q$ and $z_{n} \rightarrow z_{0}$ as $n \rightarrow \infty$ it follows that

$$
0=\lim _{n \rightarrow \infty} \Delta\left(p_{n}, z_{n}\right)=\Delta\left(q, z_{0}\right)
$$

Since for any $p \in \mathbb{T}^{3}$ the function $\Delta(p, \cdot)$ is decreasing in $(-\infty, m]$ and $q \in \partial D$ we see that $\Delta\left(q, z_{0}\right)=0$ if and only if $z_{0}=m$.

For any $q \in \partial D$ we define

$$
z(q)=\lim _{p \rightarrow q, p \in D} z(p)=m .
$$

Since the function $z(\cdot)$ is continuous on the compact set $D \cup \partial D$ and $z(q)=m$ for all $q \in \partial D$ we conclude that

$$
\operatorname{Im} z=[a, m], \quad a<m .
$$

Hence the set

$$
\left\{z \in \sigma_{t w o}(\hat{H}): z \leq m\right\}=\cup_{p \in \mathbb{T}^{3}} \sigma_{d}(h(p)) \cap(-\infty, m]
$$

coincides with the set $\operatorname{Im} z=[a, m]$. Then Lemma4.1 completes the proof of $(i i)$.

(iii) Let $\min _{p \in \mathbb{T}^{3}} \Delta(p, m) \geq 0$. Then by Lemma 3.4 for all $p \in \mathbb{T}^{3}$ the operator $h(p)$ has no eigenvalues lying on the l.h.s. of $m$. 
Hence we have

$$
\sigma(\hat{H})=[m, M]
$$

So Lemma 4.2 complete the proof of Theorem 4.3

\section{THE BIRMAN-SCHWINGER PRINCIPLE}

In this section we prove an analogue of the Birman-Schwinger principle.

For a bounded self-adjoint operator $A$, we define $n(\lambda, A)$ as

$$
n(\lambda, A)=\sup \{\operatorname{dim} F:(A u, u)>\lambda, u \in F,\|u\|=1\} .
$$

The number $n(\lambda, A)$ is equal the infinity if $\lambda$ is in the essential spectrum of $A$ and if $n(\lambda, A)$ is finite, it is equal to the number of the eigenvalues of $A$ bigger than $\lambda$. By the definition of $N(z)$ we have

$$
N(z)=n(-z,-H),-z>-\tau_{e s s}(H) .
$$

Since the function $\Delta(\cdot, \cdot)$ is positive on $\mathbb{T}^{3} \times\left(-\infty, \tau_{\text {ess }}(H)\right)$ there exists a positive square root of $\Delta(p, z)$ for any $p \in \mathbb{T}^{3}$ and $z<\tau_{\text {ess }}(H)$.

In our analysis of the spectrum of $H$ the crucial role is played by the compact operator $T(z), z<\tau_{\text {ess }}(H)$ in the space $\mathcal{H}^{(2)}$ with the entries

$$
\begin{gathered}
\left(T_{00}(z) f_{0}\right)_{0}=\left(1-u_{0}-z\right) f_{0}, \quad\left(T_{01}(z) f_{1}\right)_{0}=-\int_{\mathbb{T}^{3}} \frac{b\left(q^{\prime}\right) f\left(q^{\prime}\right) d q^{\prime}}{\sqrt{\Delta\left(q^{\prime}, z\right)}}, \\
T_{10}(z)=T_{01}^{*}(z), \quad\left(T_{11}(z) f_{1}\right)_{1}(p)=\frac{b(p)}{2 \sqrt{\Delta(p, z)}} \int_{\mathbb{T}^{3}} \frac{b\left(q^{\prime}\right) f\left(q^{\prime}\right) d q^{\prime}}{\sqrt{\Delta\left(q^{\prime}, z\right)}\left(w\left(p, q^{\prime}\right)-z\right)} .
\end{gathered}
$$

The following lemma is a realization of the well known Birman-Schwinger principle for the operator $H$ (see [4, 33, 35]).

Lemma 5.1. For $z<\tau_{\text {ess }}(H)$ the operator $T(z)$ is compact and continuous in $z$ and

$$
N(z)=n(1, T(z)) \text {. }
$$

Proof. The operator $H$ can be decomposed as

$$
H=\left(\begin{array}{ccc}
H_{00} & 0 & 0 \\
0 & H_{11} & 0 \\
0 & 0 & H_{22}
\end{array}\right)+\left(\begin{array}{ccc}
0 & H_{01} & 0 \\
H_{10} & 0 & H_{12} \\
0 & H_{21} & 0
\end{array}\right)
$$

The operator $H_{i i}-z \mathbf{I}, i=1,2$ is positive and invertible for $z<\tau_{\text {ess }}(H)$, where $\mathbf{I}$ is the identity operator on $\mathcal{H}$. Hence there exist a square root of $R_{i}(z)=\left(H_{i i}-z \mathbf{I}\right)^{-1}, i=1,2$. Then one has $f \in \mathcal{H}$ and $((H-z \mathbf{I}) f, f)<0$ if and only if $((M(z)-\mathbf{I}) g, g)>0$ and $g_{0}=f_{0}, g_{i}=\left(H_{i i}-z\right)^{\frac{1}{2}} f_{i}, i=1,2$, the operator $M(z), z<\tau_{\text {ess }}(H)$ has the entries

$$
\begin{gathered}
\left(M_{00}(z) f_{0}\right)_{0}=\left(1-u_{0}+z\right) f_{0}, M_{01}(z)=-H_{01} R_{1}^{\frac{1}{2}}(z), M_{12}(z)=-R_{1}^{\frac{1}{2}}(z) H_{12} R_{2}^{\frac{1}{2}}(z), \\
M_{10}(z)=M_{01}^{*}(z), \quad M_{21}(z)=M_{12}^{*}(z),
\end{gathered}
$$

otherwise

$$
M_{\alpha \beta}(z)=0
$$

It follows that

$$
N(z)=n(1, M(z))
$$

Let $V(z), z<\tau_{\text {ess }}(H)$ be the operator in $\mathcal{H}^{(2)}$ with the entries

$V_{11}(z)=R_{1}^{\frac{1}{2}}(z) H_{12} R_{2}(z) H_{21} R_{1}^{\frac{1}{2}}(z), \quad$ otherwise $\quad V_{\alpha \beta}(z)=M_{\alpha \beta}(z), \alpha, \beta=0,1$. 
A direct calculation shows that

$$
n(1, M(z))=n(1, V(z)) .
$$

One has $\mathcal{H}^{(2)}$ and $((V(z)-I) \varphi, \varphi)>0$ iff $\psi_{0}=\varphi_{0}$ and $\psi_{1}=R_{1}^{\frac{1}{2}}(z) \varphi_{1}$ and

$$
\begin{gathered}
\left(\psi_{0}, \psi_{0}\right)_{0}+\left(\left(H_{11}-z\right) \psi_{1}, \psi_{1}\right)_{1}<\left(M_{00}(z) \psi_{0}, \psi_{0}\right)_{0}- \\
-\left(H_{01} \psi_{1}, \psi_{0}\right)_{0}-\left(H_{10}(z) \psi_{0}, \psi_{1}\right)_{1}+\left(H_{12} R_{2}(z) H_{21} \psi_{1}, \psi_{1}\right)_{1},
\end{gathered}
$$

where $I$ is the identity operator on $\mathcal{H}^{(2)}$. This means that

$$
n(1, V(z))=n(-z, G(z))
$$

where

$$
G(z)=\left(\begin{array}{cc}
-H_{00}-2 z & -H_{01} \\
-H_{10} & H_{12} R_{2}(z) H_{21}-H_{11}
\end{array}\right)
$$

Now we represent the operator $H_{21}$ as a sum of two operators $H_{21}^{(1)}$ and $H_{21}^{(2)}$ acting from $L_{2}\left(\mathbb{T}^{3}\right)$ to $L_{2}\left(\left(\mathbb{T}^{3}\right)^{2}\right)$ as

$$
\left(H_{21}^{(1)} f_{1}\right)(p, q)=\frac{1}{2} v(p) f_{1}(q),\left(H_{21}^{(2)} f_{1}\right)(p, q)=\frac{1}{2} v(q) f_{1}(p) .
$$

Since $z<\tau_{\text {ess }}(H)$ for any $p \in \mathbb{T}^{3}$ the function $\Delta(p, \cdot)$ is positive and hence the operator $H_{11}-z-H_{12} R_{2}(z) H_{21}^{(2)}$ is positive and invertible and

$$
\left(H_{11}-z-H_{12} R_{2}(z) H_{21}^{(2)}\right)^{-\frac{1}{2}}=R_{11}^{\frac{1}{2}}(z) .
$$

Now $\psi \in \mathcal{H}^{(2)}$ and the inequality (5.4) holds iff $\eta_{0}=\psi_{0}, \eta_{1}=R_{1}^{\frac{1}{2}}(z) \varphi_{1}$ and $((T(z)-$ I) $\eta, \eta)>0$ and hence

$$
n(-z, G(z))=n(1, T(z)) .
$$

The equalities (5.2), (5.3), 5.5) and (5.6) give (5.1).

Finally we note that for any $z<\tau_{\text {ess }}(H)$ the operator $T(z)$ is compact and continuous in $z$.

\section{THE Finiteness of THE NUMBER OF EIGENVAlues OF THE OPERATOR $H$.}

Lemma 6.1. Let the conditions in part ( $i$ ) of Theorem 2.13 be fulfilled. Then for any $z \leq m$ the operator $T(z)$ is compact and is continuous from the left up to $z=m$.

Proof. Denote by $Q(p, q ; z)$ the kernel of the operator $T_{11}(z), z \leq m$, that is,

$$
Q(p, q ; z)=\frac{b(p) b(q))}{2 \sqrt{\Delta(p, z)}(w(p, q)-z) \sqrt{\Delta(q, z)}} .
$$

Since the function $v \in \mathcal{B}\left(\theta, \mathbb{T}^{3}\right)$ is even and $v(0)=0$ we have $|v(p)| \leq C|p|^{\theta}$ for any $p \in \mathbb{T}^{3}$ and for some $C>0$. By virtue of Lemmas 3.9 and A.4 the kernel $Q(p, q ; z)$ is estimated by

$$
C\left(\frac{\chi_{\delta}(p)}{|p|}+1\right)\left(\frac{|p|^{\theta}|q|^{\theta} \chi_{\delta}(p) \chi_{\delta}(q)}{p^{2}+q^{2}}+1\right)\left(\frac{\chi_{\delta}(q)}{|q|}+1\right),
$$

where $\chi_{\delta}(p)$ is the characteristic function of $U_{\delta}(0)$.

The latter function is square-integrable on $\left(\mathbb{T}^{3}\right)^{2}$ and hence we have that, for any $z \leq m$, $T_{11}(z)$ is a Hilbert-Schmidt operator.

The kernel function of $T_{11}(z)$ is continuous in $p, q \in \mathbb{T}^{3}, z<m$ and square-integrable on $\left(\mathbb{T}^{3}\right)^{2}$ for $z \leq m$. Now the continuity of the operator $T_{11}(z)$ from the left up to $z=m$ follows from Lebesgue's dominated convergence theorem. 
Since for all $z \leq m$ the operators $T_{00}(z), T_{01}(z)$ and $T_{10}(z)$ are of rank 1 and continuous from the left up to $z=m$ we can conclude that $T(z)$ is compact and continuous from the left up to $z=m$.

We are now ready for the

Proof of $(i)$ of Theorem 2.13 Let the conditions in part $(i)$ of Theorem 2.13 be fulfilled. By Lemma 5.1 we have

$$
N(z)=n(1, T(z)), \text { as } z<m
$$

and by Lemma 6.1 for any $\gamma \in[0,1)$ the number $n(1-\gamma, T(m))$ is finite. Then we have

$$
n(1, T(z)) \leq n(1-\gamma, T(m))+n(\gamma, T(z)-T(m))
$$

for all $z<m$ and $\gamma \in(0,1)$. This relation can easily be obtained by a use of the Weyl inequality

$$
n\left(\lambda_{1}+\lambda_{2}, A_{1}+A_{2}\right) \leq n\left(\lambda_{1}, A_{1}\right)+n\left(\lambda_{2}, A_{2}\right)
$$

for the sum of compact operators $A_{1}$ and $A_{2}$ and for any positive numbers $\lambda_{1}$ and $\lambda_{2}$.

Since $T(z)$ is continuous from the left up to $z=m$, we obtain

$$
\lim _{z \rightarrow m-0} N(z)=N(m) \leq n(1-\gamma, T(m)) \text { for all } \gamma \in(0,1)
$$

Thus

$$
N(m) \leq n(1-\gamma, T(m))<\infty
$$

The inequality 6.1 proves the assertion $(i)$ of Theorem 2.13

\section{ASYMPTOTICS FOR THE NUMBER OF EIGENVALUES OF THE OPERATOR $H$.}

In this section we shall closely follow the A. Sobolev method [33] to derive the asymptotics (2.5) for the number of eigenvalues of $H$.

We shall first establish the asymptotics of $n(1, T(z))$ as $z \rightarrow m-0$. Then part (ii) Theorem 2.13 will be deduced by a perturbation argument based on the following lemma.

Lemma 7.1. Let $A(z)=A_{0}(z)+A_{1}(z)$, where $A_{0}(z)\left(A_{1}(z)\right)$ is compact and continuous in $z<m$. Assume that for some function $f(\cdot), f(z) \rightarrow 0, z \rightarrow-0$ there exists the

$$
\lim _{z \rightarrow-0} f(z) n\left(\lambda, A_{0}(z)\right)=l(\lambda),
$$

and is continuous in $\lambda>0$. Then the same limit exists for $A(z)$ and

$$
\lim _{z \rightarrow-0} f(z) n(\lambda, A(z))=l(\lambda) .
$$

For the proof of Lemma7.1] see Lemma 4.9 of [33].

Remark 7.2. Since $U(\cdot)$ is continuous in $\mu>0$, according to Lemma 7.1 any perturbations of the operator $A_{0}(z)$ defined in Lemma 7.1] which is compact and continuous up to $z=m$ do not contribute to the asymptotics 2.5. During the Section 7 we use this fact without further comments.

By Assumption 2.1 we get

$$
w(p, q)=m+\frac{1}{2}\left(l_{1}(W p, p)+2 l_{2}(W p, q)+l_{1}(W q, q)\right)+O\left(|p|^{3+\theta}+|q|^{3+\theta}\right) \text { as } p, q \rightarrow 0 \text {. }
$$

By the representation 3.15 and Corollary 3.7we get

$$
\Delta(p, z)=\frac{4 \pi^{2} v^{2}(0)}{l_{1}^{3 / 2}(\operatorname{det} W)^{\frac{1}{2}}}[l(W p, p)-2(z-m)]^{\frac{1}{2}}+O\left(\left(|p|^{2}+|z-m|\right)^{\frac{1+\theta}{2}}\right)
$$


as $p,|z-m| \rightarrow 0$, where $l=\left(l_{1}^{2}-l_{2}^{2}\right) / l_{1}$.

Denote by $\hat{\chi}_{\delta}(\cdot)$ the characteristic function of $\hat{U}_{\delta}(0)=\left\{p \in \mathbb{T}^{3}:\left|W^{\frac{1}{2}} p\right|<\delta\right\}$.

Let $T(\delta ;|z-m|)$ be the operator in $\mathcal{H}^{(2)}$ defined by

$$
T(\delta ;|z-m|)=\left(\begin{array}{cc}
0 & 0 \\
0 & T_{11}(\delta ;|z-m|)
\end{array}\right),
$$

where the $T_{11}(\delta ;|z-m|)$ is the integral operator in $\mathcal{H}_{1}$ with the kernel

$$
\frac{l_{1}^{\frac{3}{2}}(\operatorname{det} W)^{\frac{1}{2}} \hat{\chi}_{\delta}(p) \hat{\chi}_{\delta}(q)(l(W q, q)+2|z-m|)^{-1 / 4}}{2 \pi^{2}(l(W p, p)+2|z-m|)^{1 / 4}\left(l_{1}(W p, p)+2 l_{2}(W p, q)+l_{1}(W q, q)+2|z-m|\right)} .
$$

The main technical point to apply Lemma 7.1 is the following

Lemma 7.3. Let the conditions in part (ii) of Theorem 2.13 be fulfilled. Then the operator $T(z)-T(\delta ;|z-m|)$ is compact and is continuous in $z \leq m$.

Proof. Applying the asymptotics (7.1, 7.2 and Lemmas 3.8 and A.4 one can estimate the kernel of the operator $T_{11}(z)-T_{11}(\delta ;|z-m|)$ by

$$
C\left[\frac{|p|^{\theta}}{|p|^{\frac{1}{2}}}+\frac{|q|^{\theta}}{|q|^{\frac{1}{2}}}+\frac{|p|^{\theta}+|q|^{\theta}}{|p|^{\frac{1}{2}}\left(p^{2}+q^{2}\right)|q|^{\frac{1}{2}}}+\frac{|m-z|^{\frac{\theta}{2}}\left(p^{2}+q^{2}\right)^{-1}}{\left(|p|^{2}+|m-z|\right)^{\frac{1}{4}}\left(|q|^{2}+|m-z|\right)^{\frac{1}{4}}}+1\right]
$$

and hence the operator $T_{11}(z)-T_{11}(\delta ;|z-m|)$ belongs to the Hilbert-Schmidt class for all $z \leq m$. In combination with the continuity of the kernel of the operator in $z<m$ this gives the continuity of $T_{11}(z)-T_{11}(\delta ;|z-m|)$ in $z \leq m$.

It is easy to see that $T_{00}(z), T_{01}(z)$ and $T_{10}(z)$ are rank 1 operators and they are continuous from the left up to $z=m$. The details are omitted.

Let us now recall some results from [33], which are important in our work. Let $\mathbf{S}_{\mathbf{r}}: L_{2}\left((0, \mathbf{r}), \sigma_{0}\right) \rightarrow L_{2}\left((0, \mathbf{r}), \sigma_{0}\right)$ be the integral operator with the kernel

$$
S(y ; t)=(2 \pi)^{-2} \frac{l_{0}}{\cosh y+s t}
$$

and

$$
\mathbf{r}=1 / 2|\log | z-m||, y=x-x^{\prime}, t=<\xi, \eta>, \xi, \eta \in \mathbb{S}^{2}, l_{0}=\left(\frac{l_{1}^{2}}{l_{1}^{2}-l_{2}^{2}}\right)^{\frac{1}{2}}, s=\frac{l_{2}}{l_{1}},
$$

$\sigma_{0}=L_{2}\left(\mathbb{S}^{2}\right), \mathbb{S}^{2}$ being the unit sphere in $\mathbb{R}^{3}$

The coefficient in the asymptotics of $N(z)$ will be expressed by means of the selfadjoint integral operator $\hat{\mathbf{S}}(\lambda), \lambda \in \mathbb{R}$, in the space $L_{2}\left(\mathbb{S}^{2}\right)$ whose kernel depends on the scalar product $t=<\xi, \eta>$ of the arguments $\xi, \eta \in \mathbb{S}^{2}$ and has the form

$$
\hat{\mathbf{S}}(t ; \lambda)=(2 \pi)^{-1} l_{0} \frac{\sinh [\lambda(\operatorname{arccoss} t)]}{\left(1-s^{2} t^{2}\right)^{\frac{1}{2}} \sinh (\pi \lambda)} .
$$

For $\mu>0$, define

$$
U(\mu)=(4 \pi)^{-1} \int_{-\infty}^{+\infty} n(\mu, \hat{\mathbf{S}}(y)) d y .
$$

Set $\mathcal{U}_{0}=U(1)$.

The following lemma can be proved in the same way as Theorem 4.5 in [33]. 
Lemma 7.4. Let $\mathbf{S}_{\mathbf{r}}$ be the operator defined in (7.3. Then for any $\mu>0$ the equality

$$
\lim _{\mathbf{r} \rightarrow \infty} \frac{1}{2} \mathbf{r}^{-1} n\left(\mu, \mathbf{S}_{\mathbf{r}}\right)=U(\mu)
$$

holds.

The following theorem is basic for the proof of the asymptotics 2.5 .

Theorem 7.5. Let the conditions in part (ii) of Theorem 2.13 be fulfilled. Then the equality

$$
\lim _{|z-m| \rightarrow 0} \frac{n(1, T(z))}{|\log | z-m||}=\lim _{\mathbf{r} \rightarrow \infty} \frac{1}{2} \mathbf{r}^{-1} n\left(1, \mathbf{S}_{\mathbf{r}}\right)
$$

holds.

Proof. As in Lemma 7.3 it can be shown that $T(z)-T(\delta ;|z-m|)$ defines a compact operator continuous in $z \leq m$ and this does not contribute to the asymptotics 2.5.

The space of functions having support in $\hat{U}_{\delta}(0)$ is an invariant subspace for the operator $T_{11}(\delta ;|z-m|)$.

Let $\hat{T}_{11}^{(0)}(\delta ;|z-m|)$ be the restriction of the operator $T_{11}(\delta ;|z-m|)$ to the subspace $L_{2}\left(\hat{U}_{\delta}(0)\right)$. One verifies that the operator $\hat{T}_{11}^{(0)}(\delta ;|z-m|)$ is unitary equivalent to the following operator $T_{11}^{(0)}(\delta ;|z-m|)$ acting in $L_{2}\left(\hat{U}_{\delta}(0)\right)$ as

$\left(T_{11}^{(0)}(\delta ;|z-m|) f\right)(p)=\frac{l_{1}^{3 / 2}}{2 \pi^{2}} \int_{U_{\delta}(0)} \frac{\left(l p^{2}+2|z-m|\right)^{-1 / 4}\left(l q^{2}+2|z-m|\right)^{-1 / 4}}{l_{1} p^{2}+2 l_{2}(p, q)+l_{1} q^{2}+2|z-m|} f(q) d q$.

Here the equivalence is performed by the unitary dilation

$$
\mathbf{Y}: L_{2}\left(U_{\delta}(0)\right) \rightarrow L_{2}\left(\hat{U}_{\delta}(0)\right), \quad(\mathbf{Y} f)(p)=f\left(U^{-\frac{1}{2}} p\right) .
$$

The operator $T_{11}^{(0)}(\delta ;|z-m|)$ is unitary equivalent to the integral operator $T_{11}^{(1)}(\delta ; \mid z-$ $m \mid): L_{2}\left(U_{r}(0)\right) \rightarrow L_{2}\left(U_{r}(0)\right)$ with the kernel

$$
\frac{l_{1}^{3 / 2}}{2 \pi^{2}} \frac{\left(l p^{2}+2\right)^{-1 / 4}\left(l q^{2}+2\right)^{-1 / 4}}{l_{1} p^{2}+2 l_{2}(p, q)+l_{1} q^{2}+2},
$$

where $r=|z-m|^{-\frac{1}{2}}$ and $U_{r}(0)=\left\{p \in \mathbb{R}^{3}:|p|<r\right\}$.

The equivalence of the operators $T_{11}^{(0)}(\delta ;|z-m|)$ and $T_{11}^{(1)}(\delta ;|z-m|)$ is performed by the unitary dilation

$$
\mathbf{B}_{r}: L_{2}\left(U_{\delta}(0)\right) \rightarrow L_{2}\left(U_{r}(0)\right), \quad\left(\mathbf{B}_{r} f\right)(p)=\left(\frac{r}{\delta}\right)^{-3 / 2} f\left(\frac{\delta}{r} p\right) .
$$

Further, we may replace

$$
\left(l p^{2}+2\right)^{-1 / 4},\left(l q^{2}+2\right)^{-1 / 4} \quad \text { and } \quad l_{1} p^{2}+2 l_{2}(p, q)+l_{1} q^{2}+2
$$

by

$$
\left(l p^{2}\right)^{-1 / 4}\left(1-\chi_{1}(p)\right),\left(l q^{2}\right)^{-1 / 4}\left(1-\chi_{1}(q)\right) \quad \text { and } \quad l_{1} p^{2}+2 l_{2}(p, q)+l_{1} q^{2},
$$

respectively, since the error will be a Hilbert-Schmidt operator continuous up to $z=m$, where $\chi_{1}(\cdot)$ is a characteristic function of the ball $U_{1}(0)$. Then we get the integral operator $T_{11}^{(2)}(r)$ on $L_{2}\left(U_{r}(0) \backslash U_{1}(0)\right)$ with the kernel

$$
l^{-\frac{1}{2}} \frac{l_{1}^{3 / 2}}{2 \pi^{2}} \frac{|p|^{-1 / 2}|q|^{-1 / 2}}{l_{1} p^{2}+2 l_{2}(p, q)+l_{1} q^{2}} .
$$


By the dilation

$$
\mathbf{M}: L_{2}\left(U_{r}(0) \backslash U_{1}(0)\right) \longrightarrow L_{2}\left((0, \mathbf{r}) \times \sigma_{0}\right),
$$

where $(M f)(x, w)=e^{3 x / 2} f\left(e^{x} w\right), x \in(0, \mathbf{r}), w \in \mathbb{S}^{2}$, one sees that the operator $T_{11}^{(2)}(r)$ is unitary equivalent to the integral operator $\mathbf{S}_{\mathbf{r}}$.

Proof of $(i i)$ of Theorem 2.13 Let the conditions in part $(i i)$ of Theorem 2.13 be fulfilled.

Similarly to [33] we can show that

$$
\mathcal{U}_{0}=U(1) \geq \frac{1}{4 \pi} \int_{-\infty}^{+\infty} n\left(1, \hat{\mathbf{S}}^{(0)}(y)\right) d y \geq \frac{1}{4 \pi} \operatorname{mes}\left\{y: \hat{S}^{(0)}(y)>1\right\},
$$

where $\hat{\mathbf{S}}^{(0)}(y)$ is the multiplication operator by the number

$$
\hat{S}^{(0)}(y)=l_{0} \frac{\sinh (\text { yarcsins })}{s y \cosh \frac{\pi y}{2}}
$$

in the subspace of the harmonics of degree zero.

The positivity of $\mathcal{U}_{0}$ follows from the facts that $l_{0}>1, \hat{S}^{(0)}(0)>1$ and continuity of $\hat{S}^{(0)}(y)$. Taking into account the inequality (7.4) and Lemmas 5.1 7.4 7.5, we complete the proof of $(i i)$ of Theorem 2.13

\section{APPENDIX A.}

Lemma A.1. Let the function $v$ as in Assumption 2.2 and the function $w$ be defined by (2.1) and $\varepsilon$ be a real-analytic conditionally negative definite function on $\mathbb{T}^{3}$ with a unique non-degenerate minimum at the origin. Then Assumption 2.5 is fulfilled

Proof. It is known that the conditionally negative definite function $\varepsilon$ admits the (LévyKhinchin) representation (see, e.g., [8])

$$
\varepsilon(p)=\varepsilon(0)+\sum_{s \in \mathbb{Z}^{d} \backslash\{0\}}\left(e^{\mathrm{i}(p, s)}-1\right) \hat{\varepsilon}(s), \quad p \in \mathbb{T}^{3},
$$

which is equivalent to the requirement that the Fourier coefficients $\hat{\varepsilon}(s)$ with $s \neq 0$ are non-positive, that is,

$$
\hat{\varepsilon}(s) \leq 0, \quad s \neq 0,
$$

and the series $\sum_{s \in \mathbb{Z}^{3} \backslash\{0\}} \hat{\varepsilon}(s)$ converges absolutely.

If the function $\varepsilon(\cdot)$ is real-valued, then the equality

$$
\hat{\varepsilon}(s)=\hat{\varepsilon}(-s), \quad s \in \mathbb{Z}^{3}
$$

holds and hence

$$
\varepsilon(p)=\varepsilon(0)+\sum_{s \in \mathbb{Z}^{3} \backslash\{0\}} \hat{\varepsilon}(s)(\cos (p, s)-1), \quad p \in \mathbb{T}^{3} .
$$

Thus $\varepsilon(\cdot)$ is even on $\mathbb{T}^{3}$.

Since $w$ and $v$ are even the function $\Lambda(\cdot)$ is also even. Then from the equality

$$
w_{0}(t)-\frac{w_{p}(t)+w_{p}(-t)}{2}=\sum_{s \in Z^{3} \backslash\{0\}} \hat{\varepsilon}(s)(1+\cos (q, s))(1-\cos (p, s))
$$


we get

$$
\Lambda(0, m)-\Lambda(p, m)=\sum_{s \in Z^{3} \backslash\{0\}}(-\hat{\varepsilon}(s))(1-\cos (p, s)) B(p, s)+\tilde{B}(p),
$$

where

$$
\begin{gathered}
B(p, s)=\frac{1}{2} \int_{\mathbb{T}^{3}} \frac{(1+\cos (q, s))\left[w_{p}(t)+w_{-p}(t)-2 m\right]}{\left(w_{p}(t)-m\right)\left(w_{-p}(t)-m\right)\left(w_{0}(t)-m\right)} v^{2}(t) d t, \\
\tilde{B}(p)=\frac{1}{4} \int_{\mathbb{T}^{3}} \frac{\left[w_{p}(t)-w_{-p}(t)\right]^{2}}{\left(w_{p}(t)-m\right)\left(w_{-p}(t)-m\right)\left(w_{0}(t)-m\right)} v^{2}(t) d t .
\end{gathered}
$$

Let $\delta>0$ such that mes $\left\{\left(\mathbb{T}^{3} \backslash U_{\delta}(0)\right) \cap \operatorname{supp} v\right\}>0$ holds. We rewrite the function $B(p, s)$ as a sum of two functions

$$
B_{1}(p, s)=\frac{1}{2} \int_{\mathbb{T}^{3} \backslash U_{\delta}(0)} \frac{(1+\cos (q, s))\left[w_{p}(t)+w_{-p}(t)-2 m\right]}{\left(w_{p}(t)-m\right)\left(w_{-p}(t)-m\right)\left(w_{0}(t)-m\right)} v^{2}(t) d t
$$

and

$$
B_{2}(p, s)=\frac{1}{2} \int_{U_{\delta}(0)} \frac{(1+\cos (q, s))\left[w_{p}(t)+w_{-p}(t)-2 m\right]}{\left(w_{p}(t)-m\right)\left(w_{-p}(t)-m\right)\left(w_{0}(t)-m\right)} v^{2}(t) d t .
$$

Since the function $w$ has a unique minimum at $(0,0)$ and $v \in \mathcal{B}\left(\theta, \mathbb{T}^{3}\right)$ we obtain that for all $p \in \mathbb{T}^{3}$ the function

$$
F(p, \cdot)=\frac{\left(1-\chi_{\delta}(\cdot)\right)\left[w_{p}(\cdot)+w_{-p}(\cdot)-2 m\right]}{\left(w_{p}(\cdot)-m\right)\left(w_{-p}(\cdot)-m\right)\left(w_{0}(\cdot)-m\right)} v^{2}(\cdot)
$$

belongs to $L_{1}\left(\mathbb{T}^{3}\right)$, where $\chi_{\delta}(\cdot)$ is the characteristic function of $U_{\delta}(0)$.

Then by the Riman-Lebesque theorem for all $p \in \mathbb{T}^{3}$ we have

$$
\int_{\mathbb{T}^{3}} \cos (t, s) F(p, t) d t \rightarrow 0 \quad \text { as } \quad s \rightarrow \infty
$$

and hence the inequalities

$$
\operatorname{mes}\left\{\left(\mathbb{T}^{3} \backslash U_{\delta}(0)\right) \cap \operatorname{supp} v(\cdot)\right\}>0
$$

and

$$
F(p, t)>0 \text { for all } p \in \mathbb{T}^{3} \text { and a.e. } t \in\left(\mathbb{T}^{3} \backslash U_{\delta}(0)\right) \cap \operatorname{supp} v(\cdot)
$$

implies that

$$
B_{1}(p, s) \rightarrow \int_{\mathbb{T}^{3}} F(p, t) d t>0 \quad \text { as } \quad s \rightarrow \infty .
$$

Then from continuity of the function

$$
\int_{\mathbb{T}^{3}} F(p, t) d t
$$

on the compact set $\mathbb{T}^{3}$ it follows that there exists $C_{1}>0$ such that $B_{1}(p, s)>C_{1}, s \in$ $\mathbb{Z}^{3}, p \in \mathbb{T}^{3}$. Since $B_{2}(p, s) \geq 0, s \in \mathbb{Z}^{3}, p \in \mathbb{T}^{3}$ we have $B(p, s)>C_{1}, s \in \mathbb{Z}^{3}, p \in$ $\mathbb{T}^{3}$. Then according to $\tilde{B}(p) \geq 0, p \in \mathbb{T}^{3}$ and $\hat{\varepsilon}(s) \leq 0, s \in \mathbb{Z}^{3} \backslash\{0\}$ (see (A.1) the representations A.3 and A.4 implies that

$$
\Lambda(0, m)-\Lambda(p, m) \geq C_{1}(\varepsilon(p)-\varepsilon(0))
$$


and since the function $\varepsilon$ has a unique non-degenerate minimum at the origin we obtain that

$$
\Lambda(0, m)-\Lambda(p, m) \geq C_{2} p^{2}
$$

for some $C_{2}>0$. The inequality A.5 completes the proof of the lemma.

Lemma A.2. Let Assumption 2.1 be fulfilled. Then there exists a $\delta$-neighborhood $U_{\delta}(0) \subset$ $\mathbb{T}^{3}$ of the point $p=0$ and a function $q_{0}(\cdot) \in C^{(2)}\left(U_{\delta}(0)\right)$ such that:

(i) for any $p \in U_{\delta}(0)$ the point $q_{0}(p)$ is a unique non-degenerate minimum of $w_{p}(\cdot)$ and

$$
q_{0}(p)=-\frac{l_{2}}{l_{1}} p+O\left(|p|^{2+\theta}\right) \text { as } p \rightarrow 0 .
$$

(ii) The function $m(\cdot)=w\left(\cdot, q_{0}(\cdot)\right)$ is of class $q_{0}(\cdot) \in C^{(3)}\left(U_{\delta}(0)\right)$ and has the asymptotics

$$
m(p)=m+\frac{l_{1}^{2}-l_{2}^{2}}{2 l_{1}}(W p, p)+O\left(|p|^{3+\theta}\right) \quad \text { as } \quad p \rightarrow 0 .
$$

Proof. (i) The existence the number $\delta>0$ and a function $q_{0}(\cdot) \in C^{(2)}\left(U_{\delta}(0)\right)$ such that for all $p \in U_{\delta}(0)$ the point $q_{0}(p)$ is the unique non-degenerate minimum point of $w_{p}(\cdot)$ can be proven by the same way as Lemma 3 in [19].

The function $q_{0}(\cdot)$ is an odd function in $p \in U_{\delta}(0)$.

Indeed, since $w(\cdot, \cdot)$ is even with respect $(p, q)$ for all $p \in U_{\delta}(0)$ we obtain

$$
w_{-p}\left(-q_{0}(p)\right)=m(p)=m(-p)=w_{-p}\left(q_{0}(-p)\right) .
$$

Since for each $p \in U_{\delta}(0)$ the point $q_{0}(p)$ is the unique non-degenerate minimum of the function $w_{p}(\cdot)$ by A.8 we have $q_{0}(-p)=-q_{0}(p), p \in U_{\delta}(0)$.

The asymptotics A.6 follows from the fact that $q_{0}(\cdot)$ is an odd function. The coefficient $-\frac{l_{2}}{l_{1}}$ is calculated using identity $\nabla w\left(p, q_{0}(p)\right) \equiv 0, p \in U_{\delta}(0)$.

(ii) By asymptotics (7.1, A.6 and the equality $m(p)=w_{p}\left(q_{0}(p)\right)$ we obtain asymptotics A.7.

Lemma A.3. If the conditions of Theorem 2.13 are fulfilled, then the operator $h(p), p \in$ $\mathbb{T}^{3}$ has no eigenvalues lying on l.h.s. of $m$. Therefore, $\inf \sigma_{\text {ess }}(H)=\inf \sigma_{\text {three }}(H)=$ $\inf \sigma_{t w o}(H)=m$.

Proof. It suffices to prove that $\inf \sigma_{t w o}(H)=m$. Let the conditions of Lemma A.3 be fulfilled. Since the function $\Delta(0, \cdot)$ is decreasing on $(-\infty, m)$ and the function $u(\cdot)$ (resp. $\Lambda(\cdot)$ ) has a unique minimum (resp. maximum) at $p=0$ for all $z<m$ and $p \in \mathbb{T}^{3}$ we have

$$
\Delta(p, z)=u(p)-z-\frac{1}{2} \Lambda(p, z)>u(0)-m-\frac{1}{2} \Lambda(0, m) .
$$

If the operator $h(0)$ has either a $m$ energy resonance or the number $z=m$ is an eigenvalue, then by Lemmas 3.2 and 3.3 we have $\Delta(0, m)=0$. Hence by inequality A.9 we conclude that $\Delta(p, z)>0$ for all $p \in \mathbb{T}^{3}$ and $z<m$. By Lemma 3.1 the operator $h(p), p \in \mathbb{T}^{3}$ has no eigenvalues lying on 1.h.s. of $m$. Thus, inf $\sigma_{t w o}(H)=m$.

Lemma A.4. Let Assumption 2.1 be fulfilled. Then there exist numbers $C_{1}, C_{2}, C_{3}>0$ and $\delta>0$ such that the following inequalities

(i) $C_{1}\left(|p|^{2}+|q|^{2}\right) \leq w(p, q)-m \leq C_{2}\left(|p|^{2}+|q|^{2}\right) \quad$ for all $p, q \in U_{\delta}(0)$,

(ii) $\quad w(p, q)-m \geq C_{3}$ for all $\quad(p, q) \notin U_{\delta}(0) \times U_{\delta}(0)$

hold. 
Proof. By Assumption 2.1 the point $(0,0) \in\left(\mathbb{T}^{3}\right)^{2}$ is the unique non-degenerated minimum of the function $w(\cdot, \cdot)$. Then by 7.1 there exist positive numbers $C_{1}, C_{2}, C_{3}$ and a $\delta$-neighborhood of $p=0 \in \mathbb{T}^{3}$ so that $(i)$ and $(i i)$ hold true.

Lemma A.5. The right-hand derivative of $D_{1}(\cdot)$ at $\zeta=0$ exists and the following equality

$$
\frac{\partial}{\partial \zeta} D_{1}(0)=2 \sqrt{2} \pi^{2} l_{1}^{-\frac{3}{2}} v^{2}(0)(\operatorname{det} W)^{-\frac{1}{2}}
$$

holds.

Proof. We consider

$$
D_{1}(\zeta)-D_{1}(0)=-\frac{\zeta^{2}}{2} \int_{U_{\delta}(0)} \frac{v^{2}(q) d q}{\left(w_{0}(0, q)+\zeta^{2}\right) w_{0}(0, q)} .
$$

The function $w_{0}(0, \cdot)$ has a unique non-degenerate minimum at $q=0$. Therefore, by virtue of the Morse lemma (see [14]) there exists a one-to-one mapping $q=\varphi(t)$ of a certain ball $W_{\gamma}(0)$ of radius $\gamma>0$ with the center at the origin to $U_{\delta}(0)$ such that:

$$
w_{0}(0, \varphi(t))=t^{2}
$$

with $\varphi(0)=0$ and for the Jacobian $J_{\varphi}(t) \in \mathcal{B}\left(\theta, U_{\delta}(0)\right)$ of the mapping $q=\varphi(t)$ the equality

$$
J_{\varphi}(0)=\sqrt{2} l_{1}^{-\frac{3}{2}}(\operatorname{det} W)^{-\frac{1}{2}}
$$

holds, where $\mathcal{B}\left(\theta, U_{\delta}(0)\right)$ can be defined similarly to $\mathcal{B}\left(\theta, \mathbb{T}^{3}\right)$.

In the integral in A.11 making a change of variable $q=\varphi(t)$ and using the equality A.12 we obtain

$$
D_{1}(\zeta)-D_{1}(0)=-\frac{\zeta^{2}}{2} \int_{W_{\gamma}(0)} \frac{v^{2}(\varphi(t)) J_{\varphi}(t)}{t^{2}\left(t^{2}+\zeta^{2}\right)} d t
$$
form

Going over in the integral in A.13 to spherical coordinates $t=r \omega$, we reduce it to the

$$
D_{1}(\zeta)-D_{1}(0)=-\frac{\zeta^{2}}{2} \int_{0}^{\gamma} \frac{F(r)}{r^{2}+\zeta^{2}} d r
$$

with

$$
F(r)=\int_{\mathbb{S}^{2}} v^{2}(\varphi(r \omega)) J_{\varphi}(r \omega) d \omega,
$$

where $\mathbb{S}^{2}$ is the unit sphere in $\mathbb{R}^{3}$ and $d \omega$ is the element of the unit sphere in this space.

Using $v, J_{\varphi} \in \mathcal{B}\left(\theta, U_{\delta}(0)\right)$ we see that

$$
|F(r)-F(0)| \leq C r^{\theta} .
$$

Indeed. Since $v, J_{\varphi} \in \mathcal{B}\left(\theta, U_{\delta}(0)\right)$ for any $p \in U_{\delta}(0)$ there exists $C>0$ such that

$$
|v(p)-v(0)| \leq C|p|^{\theta} \quad \text { and } \quad\left|J_{\varphi}(p)-J_{\varphi}(0)\right| \leq C|p|^{\theta} .
$$

Thus

$$
|F(r)-F(0)| \leq \int_{\mathbb{S}^{2}}\left|v^{2}(\varphi(r \omega))-v^{2}(0)\right|\left|J_{\varphi}(r \omega)\right| d \omega+\int_{\mathbb{S}^{2}}\left|v^{2}(0)\right|\left|J_{\varphi}(r \omega)-J_{\varphi}(0)\right| d \omega \leq C r^{\theta} .
$$


The function $D_{1}(\zeta)-D_{1}(0)$ can be rewritten in the form

$$
\begin{gathered}
D_{1}(\zeta)-D_{1}(0)=2 \sqrt{2} \pi l_{1}^{-\frac{3}{2}} v^{2}(0)(\operatorname{det} W)^{-\frac{1}{2}} \int_{0}^{\gamma} \frac{\zeta^{2} d r}{r^{2}+\zeta^{2}}+ \\
\frac{v^{2}(0)}{2} \int_{0}^{\gamma} \frac{\zeta^{2}(F(r)-F(0))}{r^{2}+\zeta^{2}} d r .
\end{gathered}
$$

By inequality A.15 we have

$$
\int_{0}^{\gamma} \frac{|F(r)-F(0)|}{r^{2}+\zeta^{2}} d r \leq C \int_{0}^{\gamma} \frac{r^{\theta}}{r^{2}+\zeta^{2}} d r .
$$

Computing the integrals

$$
\int_{0}^{\gamma} \frac{\zeta}{r^{2}+\zeta^{2}} d r \quad \text { and } \quad \int_{0}^{\gamma} \frac{\zeta r^{\theta}}{r^{2}+\zeta^{2}} d r
$$

we obtain

$$
\int_{0}^{\gamma} \frac{\zeta}{r^{2}+\zeta^{2}} d r \rightarrow \frac{\pi}{2} \text { as } \zeta \rightarrow 0+\quad \text { and } \quad \int_{0}^{\gamma} \frac{\zeta r^{\theta}}{r^{2}+\zeta^{2}} d r \rightarrow 0 \text { as } \zeta \rightarrow 0+.
$$

Hence by the equality A.16 and the inequality A.17 we have that there exists a righthand derivative of $D_{1}(\cdot)$ at $\zeta=0$ and the equality A.10 holds.

\section{Acknowledgement}

The authors would like to thank Prof. R. A. Minlos and Prof. H. Spohn and Dr.Z. I. Muminov for most stimulating discussions on the results of the paper. This work was supported by DFG 436 USB 113/4 and DFG 436 USB 113/6 projects and the Fundamental Science Foundation of Uzbekistan. The last two named authors gratefully acknowledge the hospitality of the Institute of Applied Mathematics and of the IZKS of the University of Bonn.

\section{REFERENCES}

[1] S. Albeverio, F. Gesztesy and R. Høegh-Krohn: The low energy expansion in non-relativistic scattering theory, Ann. Inst. H. Poincaré Sect. A (N.S.) 37 (1982), 1-28.

[2] S. Albeverio, R. Høegh-Krohn and T. T. Wu: A class of exactly solvable three-body quantum mechanical problems and universal low energy behavior, Phys. Lett. A 83 (1971), 105-109.

[3] S. Albeverio, S. N. Lakaev, K. A. Makarov: The Efimov Effect and an Extended Szegö-Kac Limit Theorem, Letters in Math. Phys, V. 43 (1998), 73-85.

[4] S. Albeverio, S. N. Lakaev and Z. I. Muminov: Schrödinger operators on lattices. The Efimov effect and discrete spectrum asymptotics. Ann. Henri Poincaré. 5, (2004), 743-772.

[5] Zh. I. Abdullaev, S. N. Lakaev: On the spectral properties of the matrix-valued Friedrichs model. Manyparticle Hamiltonians: spectra and scattering, 1-37, Adv. Soviet Math., 5, AMS., Providence, RI, 1991.

[6] J. I. Abdullaev, S. N. Lakaev: Asymptotics of the Discrete Spectrum of the Three-Particle Schrdinger Difference Operator on a Lattice, Theoretical and Mathematical Physics 136 (2003), No.2, 1096-1109.

[7] R. D. Amado and J. V. Noble: Efimov effect; A new pathology of three-particle Systems, II. Phys. Lett. B.35. No.1, 25-27, (1971); Phys. Lett. D.5. No.8, (1972), 1992-2002.

[8] Berg C., Christensen J. P. R, and Ressel P.: Harmonic analysis on semigroups. Theory of positive definite and related functions. Graduate Texts in Mathematics, Springer-Verlag, New York, 1984. 289 pp.

[9] G. F. Dell'Antonio, R. Figari, A. Teta: Hamiltonians for systems of $N$ particles interacting through point interactions, Ann. Inst. H. Poincaré Phys. Théor. 60 (1994), no. 3, 253-290.

[10] P. A. Faria da Veiga, L. Ioriatti and M. O'Carroll: Energy-momentum spectrum of some two-particle lattice Schrödinger Hamiltonians, Phys. Rev. E (3) 66, (2002), 016130, 9 pp.

[11] G. M. Graf and D. Schenker: 2-magnon scattering in the Heisenberg model, Ann. Inst. H. Poincaré Phys. Théor. 67 (1997), 91-107.

[12] V. Efimov: Energy levels of three resonantly interacting particles, Nucl. Phys. A 210 (1973), 157-158. 
[13] L. D. Faddeev and S. P. Merkuriev: Quantum scattering theory for several particle systems, Kluwer Academic Publishers, 1993.

[14] M. V. Fedoryuk: Asymptotics of integrals and series [in Russian], Nauka, Moscow (1987).

[15] K. O. Friedrichs: Perturbation of spectra in Hilbert space, 1965, AMS., Providence, Rhode Island.

[16] Yu. G. Kondratiev and R. A. Minlos: One-particle subspaces in the stochastic $X Y$ model, J. Statist. Phys. 87 (1997), 613-642.

[17] S. N. Lakaev: On an infinite number of three-particle bound states of a system of quantum lattice particles, Theor. and Math. Phys.89 (1991), No.1, 1079-1086.

[18] S. N. Lakaev: Some spectral properties of the generalized Friedrichs model, (Russian) Trudy Sem. Petrovsk. No. 11 (1986), 210-238, 246, 248; translation in J. Soviet Math. 45 (1989), no. 6, 1540-1565.

[19] S. N. Lakaev: Bound states and resonances fo the N-particle discrete Schrödinger operator, Theor. Math. Phys. 91 (1992), No.1, 362-372.

[20] S. N. Lakaev: The Efimov's Effect of a system of Three Identical Quantum lattice Particles, Funkcionalnii analiz i ego priloj. , 27 (1993), No.3, pp.15-28, translation in Funct. Anal.Appl.

[21] S. N. Lakaev and J. I. Abdullaev: The spectral properties of the three-particle difference Schrödinger operator, Funct.Anal. Appl. 33 (1999), No. 2, 84-88.

[22] S. N. Lakaev and T. Kh. Rasulov: Efimov's Effect in a Model of Perturbation Theory of the Essential Spectrum, Funct. Anal. Appl. 37 (2003), No. 1, 69-71.

[23] S. N. Lakaev and T. Kh. Rasulov: A Model in the Theory of Perturbations of the Essential Spectrum of Multiparticle Operators, Mathematical Notes, 73 (2003), No 3, 521-528.

[24] V. A. Malishev and R. A. Minlos: Linear infinite-particle operators. Translations of Mathematical Monographs, 143. AMS, Providence, RI.

[25] D. C. Mattis: The few-body problem on lattice, Rev.Modern Phys. 58 (1986), No. 2, 361-379.

[26] R. A. Minlos and Y. M. Suhov: On the spectrum of the generator of an infinite system of interacting diffusions, Comm. Math. Phys. 206 (1999), 463-489.

[27] R. Minlos and H. Spohn: The three-body problem in radioactive decay: the case of one atom and at most two photons, Amer. Math. Soc. Transl.(2) 177 (1996), 159-193.

[28] A. I. Mogilner: Hamiltonians of solid state physics at few-particle discrete Schrodinger operators: problems and results, Advances in Sov. Math., 5 (1991), 139-194.

[29] Yu. N. Ovchinnikov and I. M. Sigal: Number of bound states of three-particle systems and Efimov's effect, Ann. Physics, 123 (1989), 274-295.

[30] J. Rauch: Perturbation theory for eigenvalues and resonances of Schrödinger Hamiltonians, J. Funct. Anal. 35 (1980), no. 3, 304-315.

[31] M. Reed and B. Simon: Methods of modern mathematical physics. III: Scattering teory, Academic Press, N.Y., 1979.

[32] M. Reed and B. Simon: Methods of modern mathematical physics. IV: Analysis of Operators, Academic Press, N.Y., 1979.

[33] A. V. Sobolev: The Efimov effect. Discrete spectrum asymptotics, Commun. Math. Phys. 156 (1993), $127-168$.

[34] H. Tamura: The Efimov effect of three-body Schrödinger operator, J. Funct. Anal. 95 (1991), 433-459.

[35] H. Tamura: Asymptotics for the number of negative eigenvalues of three-body Schrödinger operators with Efimov effect. Spectral and scattering theory and applications, 311-322, Adv. Stud. Pure Math., 23, Math. Soc. Japan, Tokyo, 1994.

[36] X. P . Wang: On the existence of the $N$ - body Efimov effect, J. Funct. Anal. 95 (1991), 433-459.

[37] D. R. Yafaev: On the theory of the discrete spectrum of the three-particle Schrödinger operator, Math. USSR-Sb. 23 (1974), 535-559.

[38] D. R. Yafaev: Scattering theory: Some old and new problems, Lecture Notes in Mathematics, 1735. Springer-Verlag, Berlin, 2000, 169 pp.

[39] Yu. Zhukov, R. Minlos: The spectrum and scattering in the "spin-boson" model with at most three photons. Theoret. and Math. Phys. 103 (1995), no. 1, 398-411.

[40] E. A. Zhizhina: Two-particle spectrum of the generator for stochastic model of planar rotators at high temperatures, J. Statist. Phys. 91 (1998), 343-368.

[41] V. A. Zorich: Mathematical analysis I. Springer-Verlag Berlin Heildelberg 2004.

\footnotetext{
${ }^{1}$ InStitut FÜR ANGEWANDte Mathematik, Universität Bonn, WegElerstr. 6, D-53115 BonN (GERMANY)

2 SFB 611, BonN, BiBoS, BIELEFELD - BonN;
} 
3 CERFIM, LOCARNo AND ACC.ARCH,USI (SWITZERLAND) E-MAIL ALBEVERIO@UNI.BONN.DE

4 SAMarkand State University, University BouleVArd 15, 703004, SAMARKAND (UZBEKISTAN) E-MAIL: LAKAEV@YAHOO.COM

5 Samarkand State University, University Boulevard 15, 703004, SAmarkand (UZBeKISTAN) E-MAIL: RTH@MAIL.RU 